\title{
Helium exhaust experiments on JET with Type I ELMs in H-mode and with Type III ELMs in ITB discharges
}

\author{
K.-D. Zastrow ${ }^{1}$, S.J. Cox ${ }^{1}$, M.G. von Hellermann ${ }^{2}$, \\ M.G. O'Mullane ${ }^{3}$, D. Stork ${ }^{1}$, M. Brix ${ }^{4}$, C.D. Challis ${ }^{1}$, I.H. Coffey ${ }^{5}$, \\ R. Dux ${ }^{6}$, K.H. Finken ${ }^{4}$, C. Giroud ${ }^{1}$, D. Hillis ${ }^{7}$, J.T. Hogan ${ }^{7}$, \\ K.D. Lawson ${ }^{1}$, T. Loarer ${ }^{8}$, A.G. Meigs ${ }^{1}$, P.D. Morgan ${ }^{1}$, \\ M.F. Stamp ${ }^{1}$, A.D. Whiteford ${ }^{3}$ and JET EFDA Contributors ${ }^{\mathrm{a}}$ \\ ${ }^{1}$ Euratom/UKAEA Fusion Association, Culham Science Centre, Abingdon, UK \\ ${ }^{2}$ FOM Instituut voor Plasmaphysica, Associatie Euratom, Nieuwegein, The Netherlands \\ ${ }^{3}$ Department of Physics, University of Strathclyde, Glasgow, UK \\ ${ }^{4}$ Forschungszentrum Jülich GmbH, IPP, Euratom Association, Jülich, Germany \\ ${ }^{5}$ Department of Physics, Queens University, Belfast, UK \\ ${ }^{6}$ Max-Planck-Institut für Plasmaphysik, Euratom Association, Garching, Germany \\ ${ }^{7}$ Oak Ridge National Laboratory, P.O. Box 2008, Oak Ridge, USA \\ ${ }^{8}$ Association EURATOM-CEA sur la Fusion, CEA Cadarache, St Paul-lez Durance, France
}

Received 3 April 2003, accepted for publication 15 December 2004

Published 21 February 2005

Online at stacks.iop.org/NF/45/163

\begin{abstract}
An analysis of helium exhaust experiments on JET in the MkII-GB divertor configuration is presented. Helium is pumped by applying an argon frost layer on the divertor cryo pump. Measurement of the helium retention time, $\tau_{\mathrm{He}}^{*}$, is performed in two ways: by the introduction of helium in gas puffs and measurement of the subsequent decay time constant of the helium content, $\tau_{\mathrm{He}}^{\mathrm{d} *}$; and by helium beam injection and measurement of the helium replacement time, $\tau_{\mathrm{He}}^{\mathrm{r} *}$. In ELMy H-mode, with plasma configuration optimized for pumping, $\tau_{\mathrm{He}}^{\mathrm{d} *} \approx 7.2 \times \tau_{\mathrm{E}}^{\mathrm{th}}$ is achieved, where $\tau_{\mathrm{E}}^{\mathrm{th}}$ is the thermal energy replacement time. For quasi-steady internal transport barrier (ITB) discharges, the achieved $\tau_{\mathrm{He}}^{\mathrm{r} *} \approx 4.1 \times \tau_{\mathrm{E}}^{\mathrm{th}}$ is significantly lower. The achieved helium recycling coefficient, confirmed by an independent measurement to be $R_{\text {eff }} \approx 0.91$, is the same in both scenarios. None of the discharges are dominated by core confinement. The difference in $\tau_{\mathrm{He}}^{*} / \tau_{\mathrm{E}}^{\text {th }}$ is instead due to the confinement properties of the edge plasma, which is characterized by Type I ELMs for the H-mode discharges studied, and Type III ELMs for the quasi-steady ITB discharges. This difference is quantified by an independent measurement of the ratio of the helium replacement time with a helium edge source to the energy confinement time.
\end{abstract}

PACS numbers: 52.55.Fa, 52.55.Pi, 52.55.Rk, 52.25.Fi, 52.25.Vy

\section{Introduction}

Control of helium 'ash' produced in $\mathrm{D}-\mathrm{T}$ reactions is one of the key issues affecting the performance and possibly the achievable burn time of a fusion reactor. The removal of helium is determined by a combination of the intrinsic transport of helium in the plasma, especially across internal and edge transport barriers, the enrichment and compression of helium in the sub-divertor region, and the pumping and refuelling efficiency for helium. Thus, the task is one of system integration and is only partially determined by plasma physics. The overall engineering requirement is

a See annex of J. Pamela et al Fusion Energy 2002; Proc. 19th. Int. Conf. (Lyon 2002) (Vienna: IAEA). best stated [1] in terms of the ratio of the helium retention time, $\tau_{\mathrm{He}}^{*}$, to the thermal energy confinement time, $\tau_{\mathrm{E}}^{\text {th }}$, since effective $\alpha$ particle heating is essential in a burning fusion plasma. If small levels of additional impurities are present, the requirement is $\tau_{\mathrm{He}}^{*} / \tau_{\mathrm{E}}^{\text {th }} \leqslant 10$ to obtain steady-state burn conditions. With additional impurities, the requirement becomes more strict, e.g. $\tau_{\mathrm{He}}^{*} / \tau_{\mathrm{E}}^{\text {th }} \leqslant 5$ would be required if carbon concentrations were of order $3 \%$. The target for the pumping arrangement is specified in terms of the helium enrichment factor, $\eta$, i.e. the ratio of the partial pressures of helium and deuterium in the sub-divertor region (at the pump throat) to the ratio of $\mathrm{He}^{2+}$ to $\mathrm{D}^{+}$in the plasma core, which needs to be larger than 0.2 for stationary operation of ITER [2]. 
A recent review of the research on helium transport and exhaust has been written by Hogan [3]. In most experiments, helium is introduced by gas puffs and the decay time constant of the helium content, $\tau_{\mathrm{He}}^{\mathrm{d}^{*}}$, is studied. On DIII-D and JT-60U, helium neutral beam injection (NBI) was used to provide a central source [4-7] so that the replacement time, $\tau_{\mathrm{He}}^{\mathrm{r} *}$, can be measured. In L-mode and ELMy H-modes it was always found that the helium exhaust rate is limited by the pumping efficiency and not by the helium transport in the plasma edge, let alone the plasma core. The achieved ratio $\tau_{\mathrm{He}}^{*} / \tau_{\mathrm{E}}^{\text {th }}$ (measured by either of the two techniques) has been low enough and it has been shown that helium can be pumped at a satisfactory rate by divertor pumping, which is the method used in the majority of experiments, and also with pumped limiters [8].

Improved core confinement by the formation and sustainment of internal transport barriers (ITBs) is seen as a possible route to steady-state tokamak operation because of the potential of this regime for full non-inductive current drive. However, helium removal from the region confined by the ITB might not be fast enough and would thus limit the burn time. Results from experiments in JT-60U ITB discharges indicate increased helium retention in the region confined by the ITB by factors of between two and three [6], and due to reduced diffusivity by a factor of five to six [9]. Also, there is concern that, since ITB discharges to date tend to be characterized by lower edge density than ELMy Hmode plasmas, the potential for pumping of helium might be reduced. To investigate both issues, a series of experiments was conducted on JET during helium plasma operation. For comparison, we also report re-analysed results in ELMy Hmodes from earlier experiments in this paper.

In [1], two characteristic times have been introduced to quantify retention, the replacement time for a central source, $S_{0}$, defined as $\tau_{0}=N_{\mathrm{He}} / S_{0}$ and the replacement time for an edge source, $S_{\text {edge }}$, defined as $\tau_{\text {edge }}=N_{\mathrm{He}} / S_{\text {edge }} . N_{\mathrm{He}}$ is the plasma helium content that is sustained by the source in each case. In this context, the 'edge' is a region of closed flux surfaces close to the plasma boundary and should not be confused with the Scrape-Off Layer (SOL), which is a region of open field lines. Note that in this paper we use symbols different from those in [1], namely an index that refers to the location of the source (' 0 ' and 'edge'). The definition is the same.

In a numerical model both $\tau_{0}$ and $\tau_{\text {edge }}$ can be readily calculated, whereas in experiments only $\tau_{\text {edge }}$ can be measured. This is because the effect of helium recycling cannot completely be removed, so an experiment with only a central source cannot be performed. The relationship of the observed retention time to refuelling efficiency, $f$, and recycling is given by equations (5.6) and (5.10) from [1], which we reproduce here:

$$
\begin{gathered}
\tau^{*}=\tau_{0}+\tau_{\text {edge }} \frac{R_{\text {eff }}}{1-R_{\text {eff }}}, \\
R_{\text {eff }}=R_{\text {ret }} f \frac{1}{1-(1-f) R_{\text {ret }}},
\end{gathered}
$$

where $R_{\text {ret }}$ is the fraction of helium returning from the wall and divertor and $R_{\text {eff }}=\Gamma_{\text {ion }} / \Gamma_{\text {out }}$ is the fraction of the helium outflux, $\Gamma_{\text {out }}$, that is returning to the confined plasma as an edge influx, $\Gamma_{\text {ion }}$, of helium ions.
The prediction of $R_{\text {eff }}$ for ITER cannot be the subject of experimental studies on existing devices since $R_{\text {ret }}$ depends on divertor geometry and the helium pumping speed, and $f$ depends on the details of the SOL plasma. Both of these are likely to be very different, and require modelling [10] to be assessed. The goal we have set ourselves in this study is to determine the contribution of $\tau_{\text {edge }}$ to $\tau_{\mathrm{He}}^{*}$ for each operational regime, which can potentially be scaled to ITER.

In all experiments reported in this paper we make use of the helium pumping capability of the JET pumped divertor. Details on the pumping arrangement and calibration of the pumping speed for helium are given in section 2. The results on helium retention in ELMy $\mathrm{H}$-mode discharges are presented briefly in section 3. Helium ash simulation experiments in quasi-steady ITB discharges are presented in detail in section 4 and the results from all regimes are compared in section 5 by independent measurements of $R_{\text {eff }}$ and $\tau_{\text {edge }}$. We have used two different techniques to study helium retention, measurement of the time constant of the decay of the total helium content, $\tau_{\mathrm{He}}^{\mathrm{d} *}$, and measurement of the replacement time for the total helium content, $\tau_{\mathrm{He}}^{\mathrm{r} *}$. A comparison of these techniques, from a model point of view, is presented in appendix A and a discussion of statistical and systematic errors in the experiments in appendix B.

\section{Helium pumping scheme in the JET MkII-GB divertor}

All experiments presented in this paper were performed during campaigns with the JET divertor in the MkII-GB configuration [11]. Helium can be pumped by applying a layer of argon frost on the divertor cryo pump (ArFCP). The pumping speed for helium on the vessel, $S_{\mathrm{V}}(\mathrm{He})$, has been characterized as a function of various argon frost coatings using sequential helium and deuterium gas pulses. The calibration established the systematic behaviour of the pumping speed as a function of the ratio of the $\mathrm{D}_{2}+\mathrm{He}$ gas condensed on the supercritical helium cooled panels to the amount of argon frost condensed, i.e. $\mathrm{N}\left(\mathrm{D}_{2}+\mathrm{He}\right) / \mathrm{N}(\mathrm{Ar})$, termed saturation. This ratio is a function of time into each discharge and has to be evaluated to obtain the rate of helium removal during the experiment. The pumping speed for helium is a universal function of the amount of argon laid down in the most recent frosting; specifically it does not depend on the thickness of the argon layer and a refresh layer restores $S_{\mathrm{V}}(\mathrm{He})$ to its original value. The maximum values that could be achieved were $S_{\mathrm{V}}(\mathrm{He})=85-95 \mathrm{~m}^{3} \mathrm{~s}^{-1}$. For comparison, the pumping speed of the divertor cryo pump for deuterium on the JET vessel is $S_{\mathrm{V}}\left(\mathrm{D}_{2}\right)=125 \mathrm{~m}^{3} \mathrm{~s}^{-1}[12]$.

The helium pumping speed on the vessel was measured to be $S_{\mathrm{V}}(\mathrm{He})=20-55 \mathrm{~m}^{3} \mathrm{~s}^{-1}$ after a JET discharge using puffs of helium gas into the torus. These results are shown in figure 1 . The effect of loading on the argon layer did not have quite the same effect as in the earlier gas-only calibration runs, showing that the effect is dependent on the way deuterium is introduced. Specifically, the pumping speed for the same level of calculated saturation was higher after a plasma discharge than after gas-only deposition. However, no difference is seen depending on the way helium was introduced in the discharge preceding the calibration pulse, i.e. whether by gas puff or by beams. 


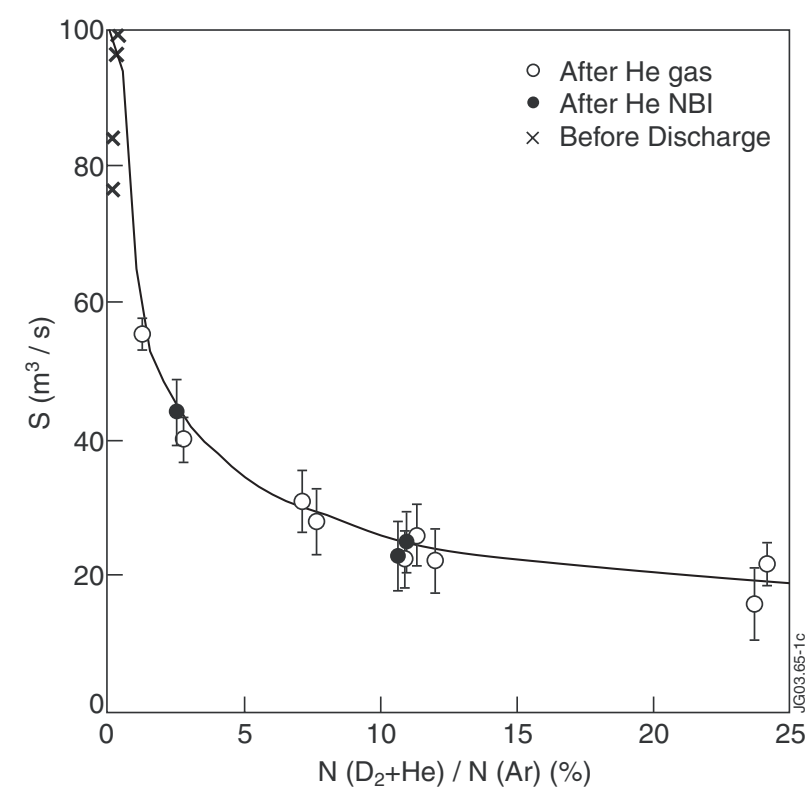

Figure 1. Pumping speed for the argon frosted divertor cryo pump on the vessel, as a function of the saturation of the argon frost layer with deuterium and helium. The pumping speed is measured using helium gas puffs into the vessel after the discharge. $\mathrm{N}\left(\mathrm{D}_{2}+\mathrm{He}\right)$ is calculated from a measurement of the sub-divertor pressures for helium and deuterium and the pumping speed of the divertor cryo pump on the sub-divertor region. $\mathrm{N}(\mathrm{Ar})$ is the amount of argon applied in the most recent frost.

After a discharge, the pumping speed can be restored by repeated argon frosting until a total of $60 \mathrm{~Pa} \mathrm{~m}^{3}$ of argon has been deposited on the pumps. At higher deposition values argon may be released, which degrades the plasma purity, and carries the risk of causing disruptions.

In figure 1 the saturation is calculated from the amount of argon applied in the most recent frost (typically $3 \mathrm{~Pa} \mathrm{~m}^{3}$ in the first frost and $1 \mathrm{Pam}^{3}$ for refresh layers), and by integrating in time the amount of helium and deuterium that was condensed on the pump. The calculation uses the time resolved measurement of the partial pressures $p$ of helium and deuterium in the sub-divertor region, obtained from Penning gauge spectroscopy $[13,14]$, and the pumping speeds $S_{\text {Div }}$ on the sub-divertor region for the two species:

$$
\begin{aligned}
N\left(\mathrm{D}_{2}+\mathrm{He}\right)= & \frac{1}{k T} \int\left(p\left(\mathrm{D}_{2}\right) \times S_{\text {Div }}\left(\mathrm{D}_{2}\right)\right. \\
& \left.+p(\mathrm{He}) \times S_{\text {Div }}(\mathrm{He})\right) \mathrm{d} t .
\end{aligned}
$$

The saturation is dominated by the amount of deuterium, since this is the majority species and because the pumping speed for deuterium is higher and not dependent on the saturation of the cryo pump. The pumping speed for helium is self-consistently calculated using the saturation as a function of time and interpolated using a cubic spline fit to the data in figure 1 (shown as a solid line) for the pumping speed itself. We use $S_{\text {Div }}\left(\mathrm{D}_{2}\right)=110 \mathrm{~m}^{3} \mathrm{~s}^{-1}$ [12] and assume $S_{\text {Div }}(\mathrm{He}) / S_{\mathrm{V}}(\mathrm{He})=110 / 125$ at all times. All measurements for partial pressures and pumping speed, as well as for the amount of argon used in the frost, are referred to room temperature [12].
Finally, we note that the mean helium removal rate of the JET ArFCP with strike points in the corner configuration is

$$
\begin{aligned}
S_{\text {Div }}(\mathrm{He}) \times p_{\text {Div }}(\mathrm{He}) & \approx 50 \mathrm{~m}^{3} \mathrm{~s}^{-1} \times 3.5 \times 10^{-3} \mathrm{~Pa} \\
& \approx 0.18 \mathrm{~Pa} \mathrm{~m}^{3} \mathrm{~s}^{-1} .
\end{aligned}
$$

Thus, the ratio of the removal rate to plasma volume $\left(S_{\text {Div }}(\mathrm{He}) \times p_{\text {Div }}(\mathrm{He}) / V_{\mathrm{P}}\right.$ with $V_{\mathrm{P}} \approx 80 \mathrm{~m}^{3}$ for JET $)$ is similar to the design basis ratio for ITER, i.e. $2.3 \times 10^{-3} \mathrm{~Pa} \mathrm{~s}^{-1}$ for JET compared to $1.8 \times 10^{-3} \mathrm{~Pa} \mathrm{~s}^{-1}$ for ITER [15].

\section{Rate of decay of helium content in Type I ELMy H-mode}

All ELMy H-mode discharges were performed at $1.94 \mathrm{~T}$, 1.9 MA and with 10-14 MW of NBI heating. The configuration chosen had an elongation of 1.68-1.74, and a triangularity of $0.26-0.30$. The discharges had Type I ELMs and achieved a thermal confinement enhancement factor as given by the $\operatorname{IPB}(98(y, 2))$ scaling law [16], of 1.1-1.3. The discharges had low $Z_{\text {eff }} \approx 2$ based on the local measurements of impurity densities ( $\mathrm{He}, \mathrm{Be}$ and $\mathrm{C}$ ) with little sign of argon. A detailed study on helium enrichment on JET, which includes some of these discharges, has previously been published by Groth et al [13]. Therefore, we only discuss the results for rate of decay of the total helium content following short $100 \mathrm{~ms}$ gas puffs, $\tau_{\mathrm{He}}^{\mathrm{d} *}$, in this section.

The achievable helium removal rate is affected by the location of the divertor strike points due to changes in the conductance for neutrals between the main chamber and the sub-divertor region [13]. To illustrate this, a comparison of a corner strike zone $(\mathrm{C})$ discharge with a vertical target strike zone (VT) discharge is shown in figure 2 . The measured decay time constant of the helium content, $\tau_{\mathrm{He}}^{\mathrm{d} *}$, is much lower in the corner configuration, due to the enhanced pumping of helium arising from the higher pressure at the pump throat. At this elevated exhausted flux, a value of $\tau_{\mathrm{He}}^{\mathrm{d} *} / \tau_{\mathrm{E}}^{\text {th }} \approx 7.2$ was achieved, well within the range required by a reactor. The decay time of the helium density was similar at all radii within the plasma. The helium profile thus relaxed in a self-similar manner with $\nabla n_{\mathrm{He}} / n_{\mathrm{He}}$ remaining approximately constant. We find that $\tau_{\mathrm{He}}^{\mathrm{d} *} / \tau_{\mathrm{E}}^{\text {th }}$ is independent, of heating power over the limited range tested. Strong deuterium gas puffing from the midplane tends to cause $\tau_{\mathrm{He}}^{\mathrm{d} *} / \tau_{\mathrm{E}}^{\text {th }}$ to increase by about $40 \%$, but this is mainly because $\tau_{\mathrm{E}}^{\mathrm{th}}$ declines as the electron density increases in the gas puff discharges, where the electron density reaches about $70 \%$ of the Greenwald density. We did not try deuterium gas puffing from the divertor during these experiments. We note that this has been found on JT-60U [7] to enhance the helium pumping rate.

The results are reproducible even across long periods of plasma operation, i.e. under different vessel conditions. We repeated discharge \#46536 in the beginning of the experiments on helium ash simulation as discharge \#53652, more than two years later, and obtained the same $\tau_{\mathrm{He}}^{\mathrm{d} *} / \tau_{\mathrm{E}}^{\mathrm{th}}$. This reproducibility is robust to the apparent difference in ELM behaviour. For \#46536 the amplitude of the $\mathrm{D} \alpha$ signal was noticeably increased following the He puff and remained so during the helium decay (see figure 2). For \#53652 the ELM amplitude did not change (see figure 16). This illustrates that some edge perturbation can be tolerated in gas puff experiments, as long as the confinement regime itself is not changed. 


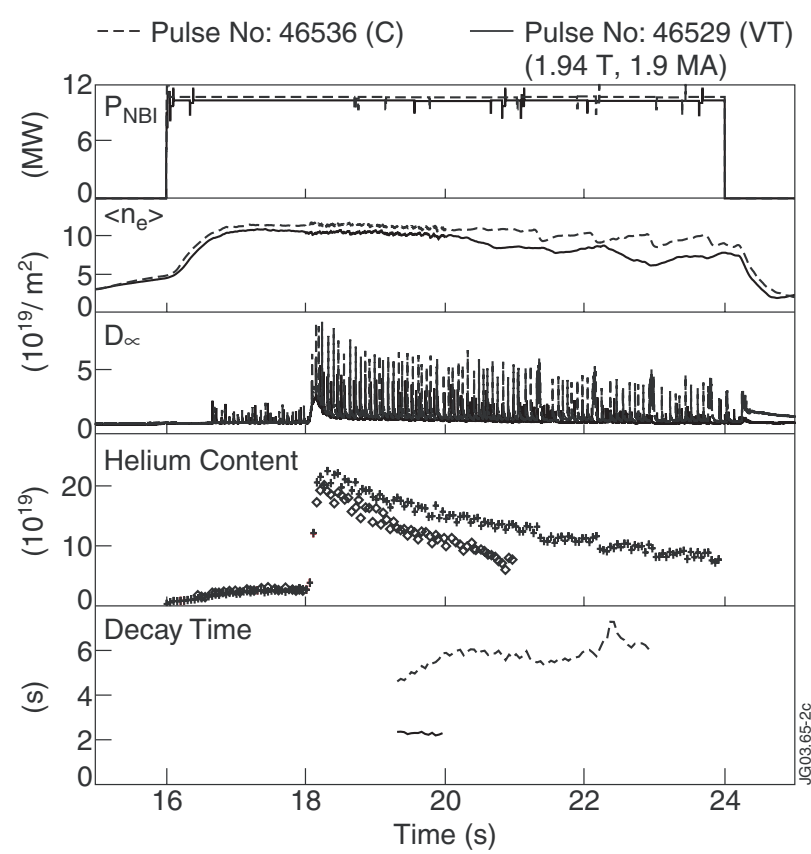

Figure 2. Comparison of the decay of the helium content for two ELMy H-mode discharges (1.94 T, 1.9 MA) at constant input power for two different strike point configurations. The discharge with the strike points in the corner ( - and $\diamond)$ exhibits a faster helium removal rate than the one with the strike points on the vertical target $(-$ - - and +).

\section{Helium ash simulation experiments in ITB discharges}

On JET it is found that impurities accumulate in the region confined by the ITB in discharges with large values of $\beta_{\mathrm{N}}$ (strong ITBs) at high magnetic field (see [17] and references therein) with higher $Z$ impurities exhibiting the strongest peaking. This has been explained by a reduction of the turbulence driven diffusion coefficient, $D$, in the presence of inward convection, $v$, where the latter was found to be in agreement with neo-classical predictions. As shown in figure 3, helium is also subject to this accumulation. The helium density in the region confined by the ITB (shown at $R=3.14$ and $3.32 \mathrm{~m}$ ) increases while it decreases outside the ITB footpoint (shown at $R=3.74$ and $3.81 \mathrm{~m}$ ). While this is interesting in its own right, it does not answer the question of what the ratio of helium replacement time to thermal energy confinement time, $\tau_{\mathrm{He}}^{\mathrm{r} *} / \tau_{\mathrm{E}}^{\mathrm{th}}$, would be with a central source under these conditions. The reduced diffusion, which acts on the gradient, can easily result in strongly peaked density profiles with a central source (see appendix A), but then again the same reduction in turbulence will be responsible for the improved energy confinement time, and it is only the ratio of these that we are concerned with. If the peaking is mainly due to inward convection, this may not be very efficient at retaining a central source since it acts on the density (see appendix A).

The more radial structure there is in the transport coefficients, the more important it becomes to conduct experiments that measure the replacement time with a central source rather than the decay time of the helium content after a puff (see appendix A). In addition, ITB discharges on JET rely on a low edge density, and therefore we find that, when helium

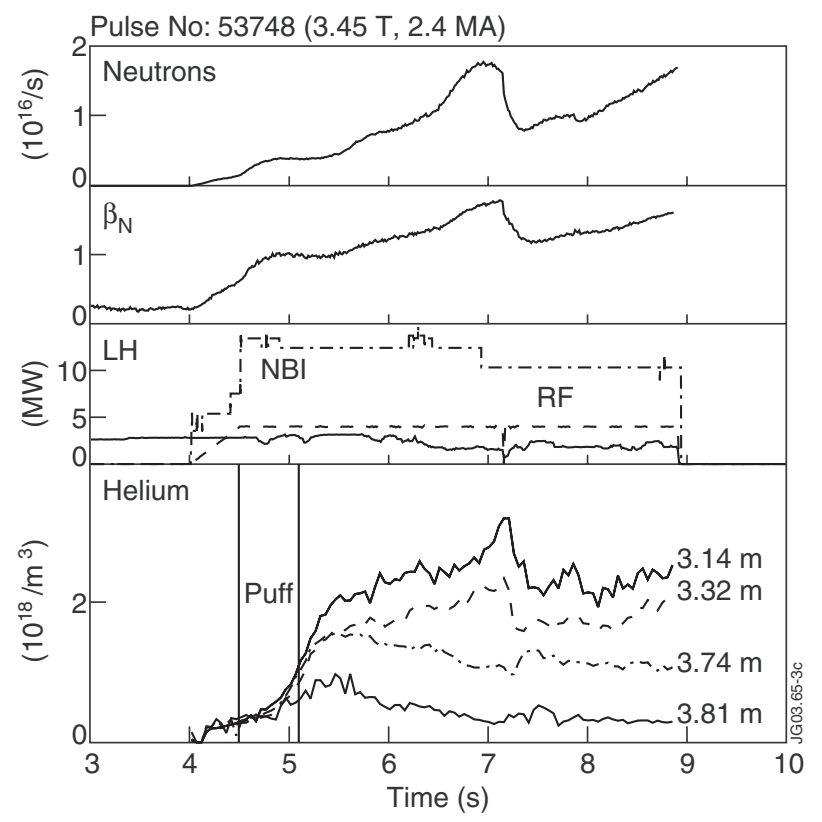

Figure 3. Helium is slowly introduced by gas puff into a discharge with reversed $q$-profile prior to ITB formation. Helium is not pumped (but there is good potential for pumping, i.e. $\eta=0.79 \pm 0.30)$. The observed changes in the helium density profile reveal changes in $v / D$, as shown in the bottom part of the figure (two chords with radii enclosed by the ITB and two chords with radii outside the ITB footpoint are shown). ITBs are first formed on the reversed $q$ profile region $(t=5.5 \mathrm{~s})$. The first strong ITB is located at $q=3(t=6.5 \mathrm{~s})$. To avoid the $\beta$ limit, the power is stepped down $(t=6.9 \mathrm{~s})$, the subsequent loss of the ITB results in a redistribution of helium. The second strong ITB is located at $q=2(t=8.1 \mathrm{~s})$.

is puffed in the same way as in ELMy H-mode discharges (see section 3), a back transition is triggered due to the increased edge electron density. The helium retention time in ITB discharges was, therefore, studied using helium neutral beams to provide a central source, to simulate the production of helium ash in a burning core. To this end, one of the two JET beam systems was converted to helium beams with an injection energy of $70 \mathrm{keV}$. For the discharges in this study, $\approx 60 \%$ of the helium is deposited within $r / a \approx 0.4$, i.e. within the region enclosed by core ITBs.

For this paper we chose to study two different ITB scenarios, as illustrated in figures 4-6, at 2.63 T/2.2 MA and at 3.45 T/2.4 MA. The latter is a quasi-steady-state ITB scenario where lower hybrid current drive and heating (LHCD) was used to slow down the current profile evolution. Both make use of a LHCD prelude phase to create a reversed $q$-profile at the onset time of the main heating [18]. Since helium beams have a larger shine through, it was necessary to develop scenarios with higher line average density than is normally the case on JET. The observed dynamics are very similar, and there are no fundamental differences between these discharges and more typical ITB discharges. Weak ITBs are formed in the region of the reversed $q$-profile for all these discharges, and in addition stronger ITBs are formed when the minimum in the $q$-profile reaches rational values, but these collapse quickly as the $q$-profile continues to evolve (see figure 6). At least $14 \mathrm{MW}$ of beam power is required for strong wide ITBs, as shown in figure 3, and they were therefore not accessible (see [19] 


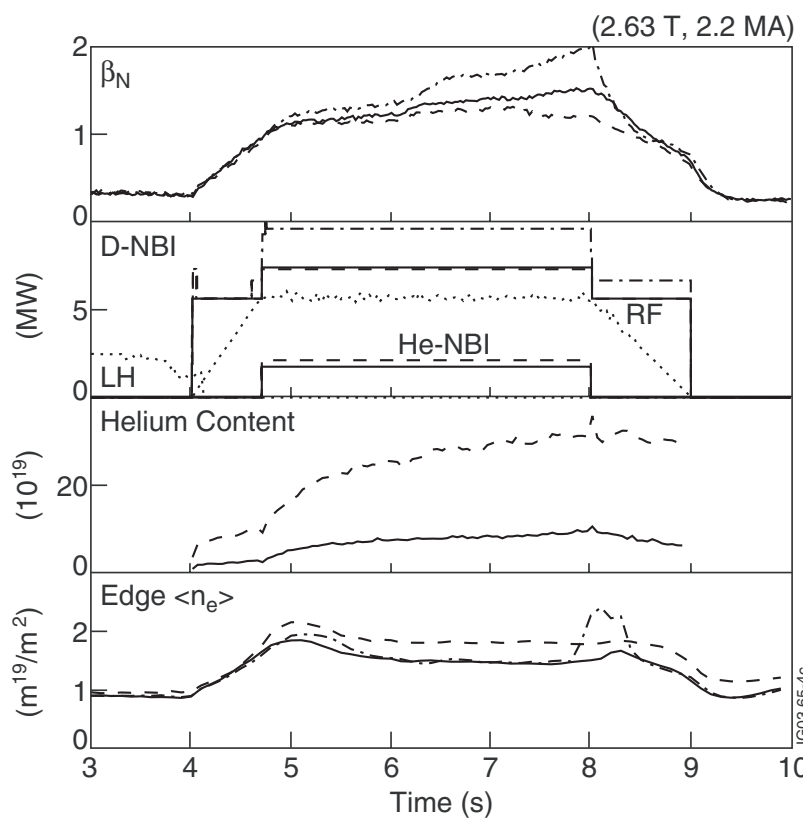

Figure 4. Comparison of three ITB discharges with identical current profile evolution and heating power (LH and RF heating are only shown once for clarity; $\cdots \cdots)$. With $8 \mathrm{MW}$ of $\mathrm{D}$ beams and $\approx 2 \mathrm{MW}$ of helium beams, but without helium pumping (- - - ), the edge density is increased compared to the D only reference pulse $(-\cdot-)$. With helium pumping $(-)$, the edge density is controlled, and a reduction of the helium core concentration from $20 \%$ to $6 \%$ is achieved. The discharge does not reach the same value of $\beta_{\mathrm{N}}$ as the reference pulse, because of the larger shine through of helium beams compared to deuterium beams.

for a detailed discussion of the requirements on JET for ITB formation on the one hand and access to high performance ITBs on the other).

Quasi-steady-state helium exhaust is provided by the ArFCP for the whole time, although the reduction of the helium pumping speed becomes noticeable towards the end of the heating phase, and as a consequence the derived helium replacement time increases (illustrated in figure 5 by the rising helium density in the core). In addition, the use of the ArFCP provides effective edge density control by reducing the helium recycling flux, as shown in figure 4 , for up to $5 \mathrm{~s}$ with $2 \mathrm{MW}$ of helium beam power, and up to $3 \mathrm{~s}$ with $3 \mathrm{MW}$. The discharges exhibit Type III ELMs throughout the helium beam injection phase. For the two discharges with helium beams in figure 4, the helium enrichment factor $\eta$ is $0.58 \pm 0.28$ without the ArFCP, and $0.42 \pm 0.20$ with ArFCP. These values demonstrate that the low edge and SOL density of ITB discharges does not impede helium removal. The discharge in figure 4 with ArFCP has the best value for $\tau_{\mathrm{He}}^{\mathrm{r} *} / \tau_{\mathrm{E}}^{\text {th }}=5$ that was obtained at $2.63 \mathrm{~T} / 2.2 \mathrm{MA}$, because of its improved energy confinement as indicated by its value of $\beta_{\mathrm{N}}=1.4$.

The results obtained in a scan of the helium source rate at otherwise constant time evolution in the two ITB scenarios (as shown in figures 4 and 5 , respectively) is shown in figure 7. The figure shows $\tau_{\mathrm{He}}^{\mathrm{r} *} / \tau_{\mathrm{E}}^{\mathrm{th}}$, the achieved helium enrichment and, for reference, the corresponding values of $\beta_{\mathrm{N}}$, averaged during the phase when the helium content was in steady state. The helium source rate was varied by using one, two or three helium sources (positive ion neutral injectors; PINIs), respectively.

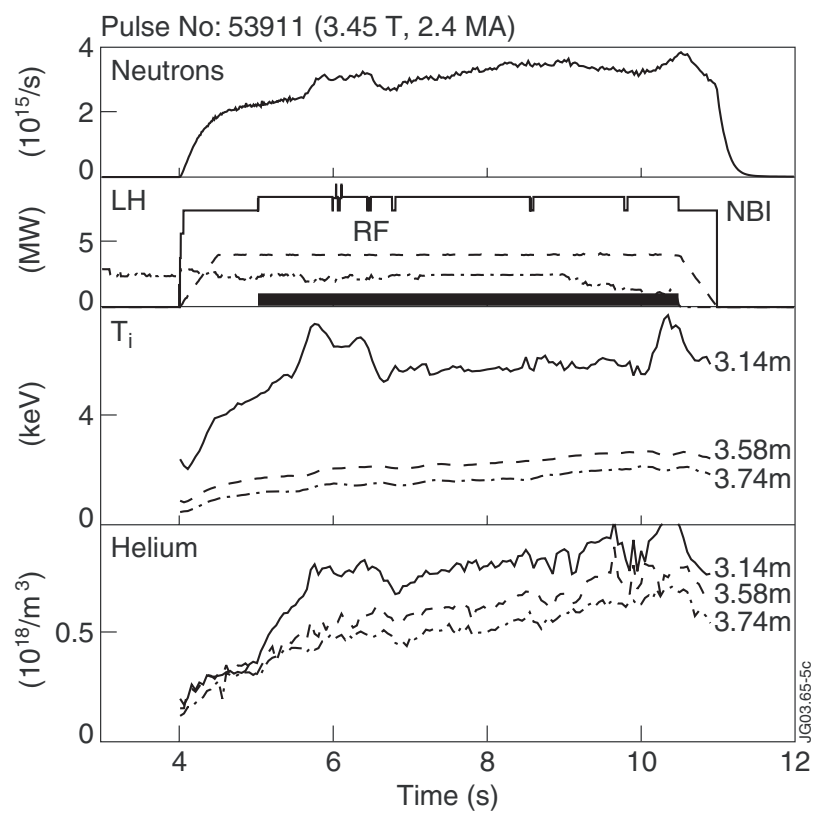

Figure 5. ITB discharge with identical current profile evolution and heating power as the discharge shown in figure 3 up to $=4.8 \mathrm{~s}$. With $1 \mathrm{MW}$ of helium beams (black bar), the edge density (not shown) is controlled for $5 \mathrm{~s}$, while the pumping speed for helium decreases during this time as indicated by the slight increase of the helium density in the core. The formation of the $q=3$ ITB at $t=5.6 \mathrm{~s}$ and the $q=2$ ITB at $t=10.0 \mathrm{~s}$ and their collapse can be clearly observed in the neutron yield, central ion temperature and helium density profile. In between these events, ITBs form and collapse rapidly in the region of the plasma where the $q$-profile is reversed, which is the reason for the observed fluctuations on neutron yield, central ion temperature and to some extent central helium content. Radial profiles for this discharge are shown in figure 6.

The highest helium source rate $\left(2.3 \times 10^{20} \mathrm{~s}^{-1}\right)$ is equivalent to that produced by $130 \mathrm{MW}$ of $\alpha$ heating, i.e. a total fusion power of $660 \mathrm{MW}$.

\section{Comparison of helium retention in ELMy H-mode and ITB discharges}

The database for the regime comparison consists of discharges with both experimental techniques ( $\tau_{\mathrm{He}}^{\mathrm{d} *}$ for ELMy H-modes, $\tau_{\mathrm{He}}^{\mathrm{r} *}$ for ITB discharges). The systematic differences expected for these two methods in identical plasma conditions is discussed in appendix A. We expect $\tau_{\mathrm{He}}^{\mathrm{d} *}<\tau_{\mathrm{He}}^{\mathrm{r} *}$. This means that, if $\tau_{\mathrm{He}}^{\mathrm{d} *} / \tau_{\mathrm{E}}^{\text {th }}$ was lower in ELMy H-mode than $\tau_{\mathrm{He}}^{\mathrm{r} *} / \tau_{\mathrm{E}}^{\text {th }}$ in ITB discharges, the difference could be due to the experimental technique. In contrast, we find that the lowest $\tau_{\mathrm{He}}^{\mathrm{d} *} / \tau_{\mathrm{E}}^{\text {th }}$ that was achieved in ELMy H-mode discharges is significantly larger than the lowest $\tau_{\mathrm{He}}^{\mathrm{r} *}<\tau_{\mathrm{He}}^{\mathrm{r} *}$ in ITB discharges. Note that from here on we will only refer to the helium retention time $\tau_{\mathrm{He}}^{*}$ for simplicity.

The results are shown in figure 8 where $\tau_{\mathrm{He}}^{*} / \tau_{\mathrm{E}}^{\text {th }}$ and the helium enrichment factor are plotted against edge plasma density for all discharges, including variation in ICRH and helium beam heating power beyond the range selected for figure 7. The figure contains only discharges with an optimized strike point configuration for pumping, i.e. a corner configuration. ELMy H-mode discharges in the vertical target 

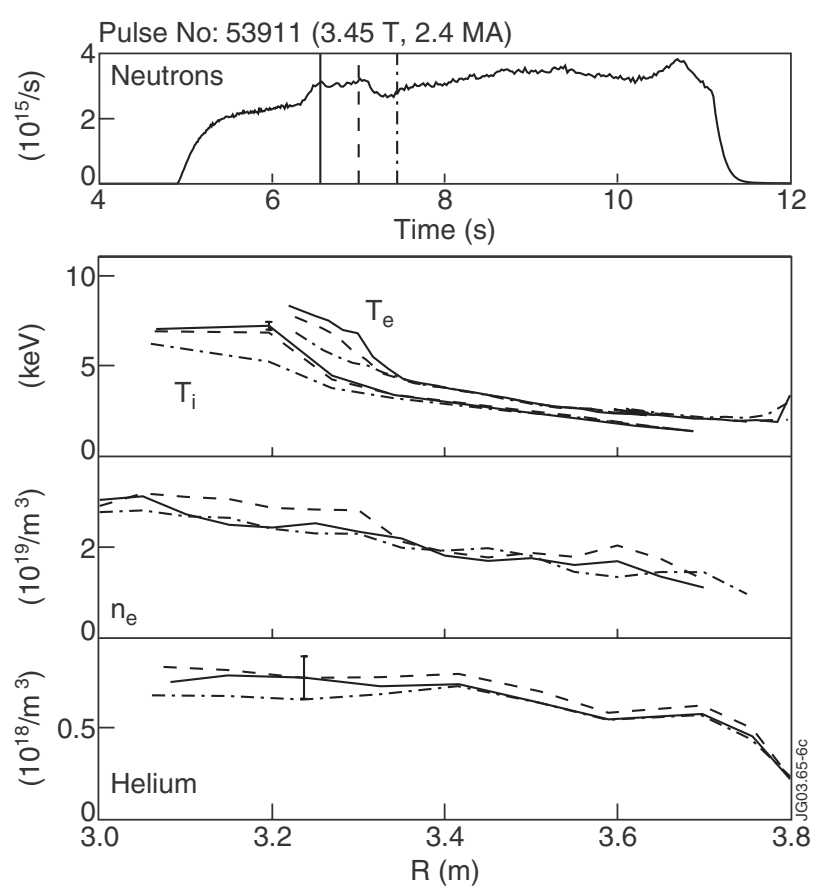

Figure 6. Radial profiles of electron and ion temperature, as well as electron and helium density, for the discharge shown in figure 5 . The ITB is located in the core $(\approx 3.3 \mathrm{~m})$ and does not expand to large radii. The magnetic axis is at $R=3.0 \mathrm{~m}$ for this shot. The panel at the top is reproduced from figure 5 to indicate the time of the profiles relative to the time evolution of the discharge.

configuration have been excluded. ITB discharges were only performed in the corner configuration. Also shown in figure 8 is the thermal confinement enhancement factor as given by the $\operatorname{IPB}(98(y, 2))$ scaling law [16]. This demonstrates that the low edge confinement in these particular ITB discharges is not offset by an increased core confinement.

In equation (1) there are three terms that could potentially differ between these regimes, namely the retention time for a central source $\tau_{0}$, the retention time for an edge source $\tau_{\text {edge }}$, and the effective recycling coefficient $R_{\text {eff }}$. We cannot measure $\tau_{0}$ directly, but we can measure the other two terms, which we elaborate in the following.

First, taking a time $t<t_{0}$ before the helium puff or before the start of helium beam injection when the total helium content, $N_{\mathrm{He}}$, is in steady state (see appendix B), we can determine the total helium influx under the assumption of toroidal and poloidal symmetry from

$$
\begin{gathered}
\tau_{\text {edge }}=\frac{N_{\mathrm{He}}}{\Gamma_{\text {edge }}}, \\
\Gamma_{\text {edge }}=2 \pi A_{\mathrm{P}} I_{30.4}\left(\frac{S}{X B}\right)_{\mathrm{He}^{+1}, 30.4} .
\end{gathered}
$$

Here $I_{30.4}$ is the line of sight integrated intensity, measured by a vacuum UV spectrometer with a line of sight located in the geometric midplane of the vessel, of the $\mathrm{He}^{+1}$ line at $\lambda=30.4 \mathrm{~nm}$, absolutely calibrated in $\mathrm{ph} /\left(\mathrm{m}^{2} \mathrm{sr} \mathrm{s}\right) . A_{\mathrm{P}}$ is the plasma surface area and $S / X B$ is the measure of ionizations per photon [20] for this spectral line. The coefficient is evaluated for each discharge using atomic data taken from

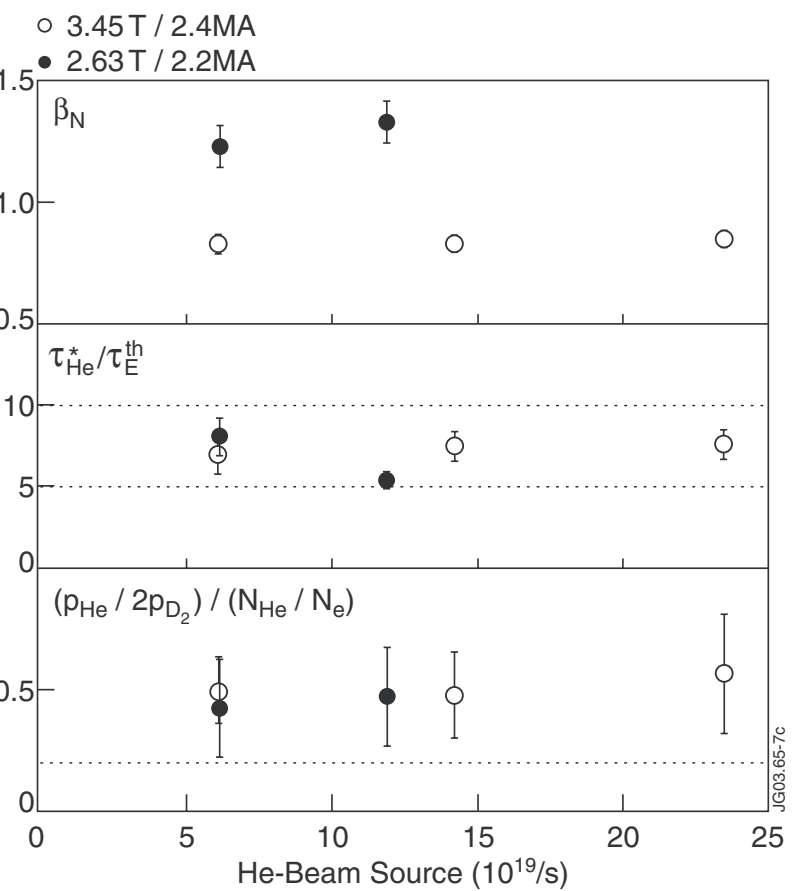

Figure 7. Overview of results, averaged during the phase in each discharge when the helium content is in steady state, for $\tau_{\mathrm{He}}^{\mathrm{r} *} / \tau_{\mathrm{E}}^{\text {th }}$ and helium enrichment factor $\eta$ with helium pumping for two reversed $q$-profile scenarios. The best value for $\tau_{\mathrm{He}}^{\mathrm{r} *} / \tau_{\mathrm{E}}^{\mathrm{th}}$ was obtained for the $2.63 \mathrm{~T} / 2.2 \mathrm{MA}$ discharge shown in figure 3 , because of its improved energy confinement as indicated by its value of $\beta_{\mathrm{N}}=1.4$. The heating power to form an ITB with the values of $\beta_{\mathrm{N}}$ at 3.45 T/2.4 MA as in figure 3 while retaining edge density control was not available due to the conversion of half the beams to helium, and only core ITBs were obtained as shown in figures 5 and 6.

ADAS [21], and turns out to be a weak function of density and temperature, with an average value of about 1.15 for the plasma conditions in the edge of JET.

Active pumping of helium is crucial in order to make this measurement, since without it the helium level would not be in steady state. In the following, we make the assumption that $\tau_{\text {edge }}$ does not change after the gas puff or during helium beam injection. In the case of the ITB discharges this assumption is probably incorrect, since the total heating power also increases when the helium beams are injected, see figures 3-5. For the example of the discharge shown in figure 3 , where we can measure $\tau_{\text {edge }}$ throughout the whole time evolution, we find that $\tau_{\text {edge }} / \tau_{\mathrm{E}}^{\mathrm{th}}$ decreases from an initial value of about $\approx 0.25$ (before the formation of the strong barrier) to $\approx 0.10$ at the peak of the neutron yield.

As defined in the introduction, $R_{\mathrm{eff}}=\Gamma_{\text {ion }} / \Gamma_{\text {out }}$ is the fraction of the helium outflux, $\Gamma_{\text {out }}$, that is returning to the confined plasma as an edge influx, $\Gamma_{\text {ion }}$, of helium ions. This can be measured independently, since we can make a measurement of $\Gamma_{\text {ion }}$, again based on the intensity of the $\mathrm{He}^{+1}$ line at $30.4 \mathrm{~nm}$ but this time after the gas puff or during helium beam injection. In addition, we can perform a calculation of the rate at which helium is pumped, $\Gamma_{\text {out }}$, considering two terms representing the wall and the ArFCP, thus

$$
R_{\text {eff }}=\frac{\Gamma_{\text {ion }}}{\Gamma_{\text {out }}}=\frac{\Gamma_{\text {edge }}}{\Gamma_{\text {edge }}+\Gamma_{\text {pump }}}=\frac{\Gamma_{\text {edge }}}{\Gamma_{\text {edge }}\left(1+\varepsilon_{\text {Wall }}\right)+\Gamma_{\text {ArFCP }}} .
$$




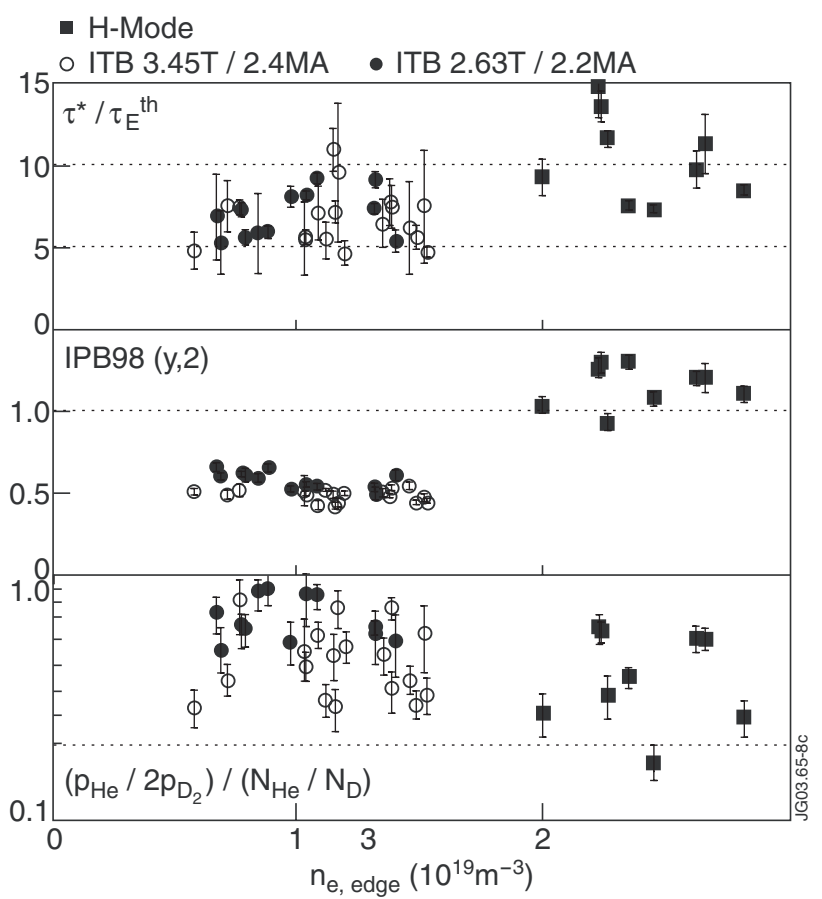

Figure 8. Overview of results for $\tau_{\mathrm{He}}^{*} / \tau_{\mathrm{E}}^{\text {th }}$ and helium enrichment factor as function of edge density. Also shown is the confinement enhancement factor. Up to three data points are taken in each discharge, and results averaged during $0.5 \mathrm{~s}$. The error bar reflects the variation of the data within each $0.5 \mathrm{~s}$ interval. All results are obtained with plasma configuration optimized for pumping, i.e. strike points in the corner on the horizontal target of the Mark II-GB divertor, but at varying pumping speed due to variations in the saturation of the ArFCP.

Table 1. Constants quantifying the helium exhaust for the four regimes studied in this paper. The solid lines in figure 9 and the $x$-axis for the data in figure 10 are calculated using these coefficients.

\begin{tabular}{lll}
\hline & $\varepsilon_{\text {Wall }}$ & $\left\langle\tau_{\text {edge }} / \tau_{\mathrm{E}}^{\text {th }}\right\rangle$ \\
\hline L-mode (1.94 T/1.9 MA) & 0.011 & 0.50 \\
ELMy H-mode (1.94 T/1.9 MA) & 0.017 & 0.92 \\
ITB at (2.63 T/2.2 MA) & 0.015 & 0.44 \\
ITB at (3.45 T/2.4 MA) & 0.024 & 0.58 \\
\hline
\end{tabular}

The rate $\Gamma_{\text {ArFCP }}$ of helium pumped by the ArFCP can be determined from the sub-divertor partial pressure of helium and the pumping speed for helium (see section 2). The fraction of helium pumped by the wall, defined here as $\varepsilon_{\text {Wall }}=$ $\Gamma_{\text {Wall }} / \Gamma_{\text {edge }}$, can be estimated from the reference discharges that we performed for each scenario without active pumping, i.e. for $\Gamma_{\mathrm{ArFCP}}=0$, making the assumption that $\tau_{0} / \tau_{\mathrm{E}}^{\text {th }}=1$ and using the measurement of $\tau_{\text {edge }}$ from equation (6). Note that using this definition, $\varepsilon_{\text {Wall }}$ is related to a measurement of $R_{\text {eff }}$ and, therefore, $\left(1-\varepsilon_{\text {Wall }}\right) \neq R_{\text {ret }}$; see equation (2). Specifically, $\varepsilon_{\text {Wall }}$ is not only a property of the wall, but also includes the properties of the SOL plasma. One consequence of this is that the result differs for each regime studied; see table 1. The result is not sensitive to the assumption for $\tau_{0} / \tau_{\mathrm{E}}^{\text {th }}=1$ since the decay or replacement time without active pumping is in the range $\tau_{\mathrm{He}}^{*} / \tau_{\mathrm{E}}^{\text {th }} \approx 20-80 \gg 1$.

We can now combine all measurements in a single plot of $\tau_{\mathrm{He}}^{*} / \tau_{\mathrm{E}}^{\text {th }}$ against $R_{\text {eff }}$, and this is shown as figure 9. The solid

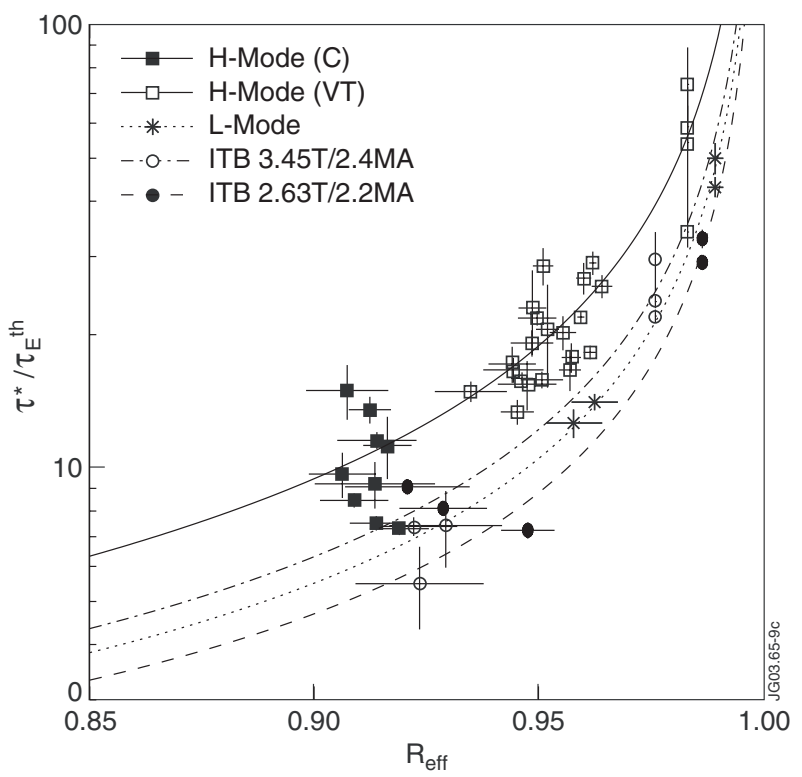

Figure 9. Overview of results for $\tau_{\mathrm{He}}^{*} / \tau_{\mathrm{E}}^{\text {th }}$ plotted against the independent estimate of effective recycling coefficient as obtained from a measurement of the influx and a calculation of the pumping rate, including removal by the ArFCP and by wall pumping. The curves represent $1+\left\langle\tau_{\text {edge }} / \tau_{\mathrm{E}}^{\text {th }}\right\rangle \times R_{\text {eff }} /\left(1-R_{\text {eff }}\right)$ using the average of the measured $\tau_{\text {edge }} / \tau_{\mathrm{E}}^{\text {th }}$ for each of the regimes, see table 1 . $\mathrm{H}$-mode data follow the same curve for corner $(\mathrm{C})$ and vertical target (VT) configuration. Only ITB discharges with one helium PINI are included in this figure. Data from discharges with two or more helium PINIs all lie significantly below the corresponding curve and are not shown, as explained in the text.

lines represent $1+\left\langle\tau_{\text {edge }} / \tau_{\mathrm{E}}^{\text {th }}\right\rangle \times R_{\text {eff }} /\left(1-R_{\text {eff }}\right)$ where $\left\langle\tau_{\text {edge }} / \tau_{\mathrm{E}}^{\text {th }}\right\rangle$ is the ensemble average for each of the four regimes; see table 1 . All curves are made to pivot around the average $\tau_{\text {edge }} / \tau_{\mathrm{E}}^{\text {th }}$ for the shot with wall pumping, which is the one with the highest value for $R_{\text {eff }}$ for each group. The observed trend for ELMy H-mode and L-mode discharges with active pumping is well reproduced by the curve. Specifically for ELMy H-mode discharges, data with corner configuration and vertical target configuration follow the same trend, the difference in helium exhaust between them being explained by the independent measurement of a different $R_{\text {eff }}$.

The L-mode discharges (the data are based on a measurement of $\tau_{\mathrm{He}}^{\mathrm{d} *}$ the same as for ELMy H-mode) were performed in vertical target configuration. During earlier studies in JET with the Mark I divertor a pair of discharges with optimized pumping, one in H-mode and one in L-mode, was performed with $\tau_{\mathrm{E}}(\mathrm{H})=1.8 \times \tau_{\mathrm{E}}(\mathrm{L})$ and $\tau_{\mathrm{He}}^{\mathrm{d} *}(\mathrm{H})=$ $3.8 \times \tau_{\mathrm{He}}^{\mathrm{d} *}(\mathrm{~L})[3,22]$ which is in qualitative agreement with our result that $\tau_{\text {edge }}(\mathrm{H})=1.8 \times \tau_{\text {edge }}(\mathrm{L})$.

For ITB discharges, the trend is less well reproduced, and only discharges with just one helium beam source are close to the trend line. Data with two or more PINIs (not shown in figure 9) lie well below the trend lines. This is probably due to a reduction of $\tau_{\text {edge }} / \tau_{\mathrm{E}}^{\text {th }}$ caused by the increase in heating power, rather than an error in the measurement of $R_{\text {eff }}$. If we assume that $\tau_{\text {edge }} / \tau_{\mathrm{E}}^{\text {th }}$ can be as low as 0.10 (as obtained for the discharge shown in figure 3 ) we could explain all data. With this in mind we can conclude that the best ITB discharges have a much lower $\tau_{\mathrm{He}}^{*} / \tau_{\mathrm{E}}^{\text {th }}$ than the best ELMy H-mode discharges 


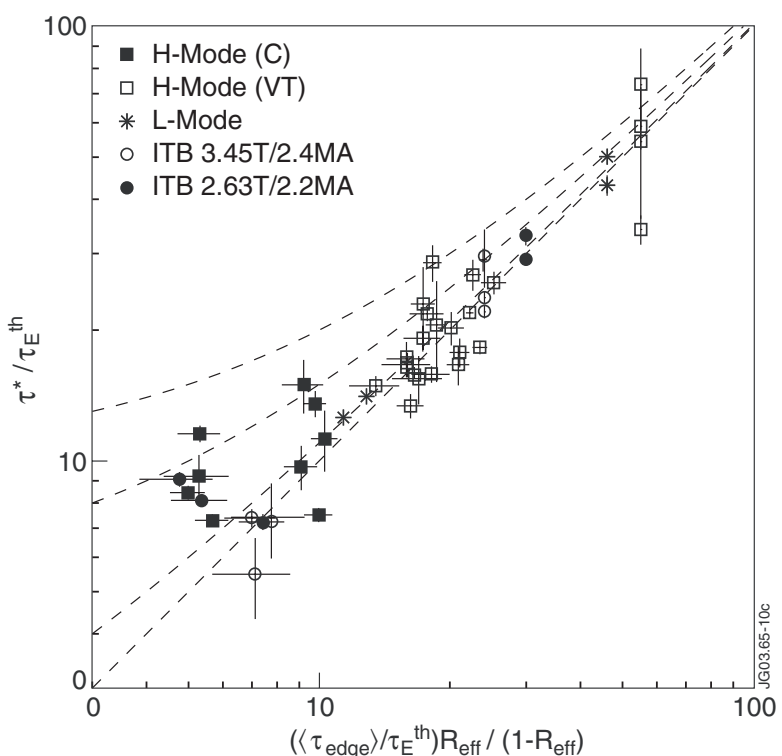

Figure 10. As figure 8 except the $x$-axis is the product of the ensemble average $\left\langle\tau_{\text {edge }} / \tau_{\mathrm{E}}^{\text {th }}\right\rangle$ and $R_{\text {eff }} /\left(1-R_{\text {eff }}\right)$ instead of $R_{\text {eff }}$. Also shown is one curve to represent unity as well as three curves with an offset due to three assumptions, $\tau_{0} / \tau_{\mathrm{E}}^{\text {th }}=1,5$ and 10 , respectively.

because of the lower value for $\left\langle\tau_{\text {edge }} / \tau_{\mathrm{E}}^{\text {th }}\right\rangle$ that characterizes these regimes.

We can, therefore, take the next step and plot all measurements against $\left\langle\tau_{\text {edge }} / \tau_{\mathrm{E}}^{\text {th }}\right\rangle \times R_{\text {eff }} /\left(1-R_{\text {eff }}\right)$, in figure 10 . The four curves in this figure represent unity, and three separate curves for different values of $\tau_{0} / \tau_{\mathrm{E}}^{\text {th }}$ (e.g. 1, 5 and 10). Although all measurements follow the trend reasonably well, over almost two orders of magnitude, it is not possible to conclude what $\tau_{0} / \tau_{\mathrm{E}}^{\text {th }}$ is for each of the regimes because the scatter is too large. Also, it is worthwhile recalling that the data for L-mode and ELMy H-mode discharges have been obtained from a measurement of the rate of decay of the total helium content and cannot be extrapolated to $R_{\text {eff }}=0$ even if the scatter was lower, as explained in appendix A.

Finally, we note that the agreement between data and prediction using the zero-dimensional model in figures 9 and 10 is only sensitive to errors in the relative calibration between the measurement of the helium content and the pumping rate. Specifically, the absolute calibration of the $\mathrm{He}^{+1}$ line at $\lambda=30.4 \mathrm{~nm}$ line cancels. This can be seen when equations (1) and (5) to equation (7) are combined

$$
\tau^{*}=\tau_{0}+\tau_{\text {edge }} \frac{\Gamma_{\text {edge }}}{\Gamma_{\text {pump }}}=\tau_{0}+\frac{N_{\mathrm{He}, t<t_{0}}}{\Gamma_{\text {pump }}} \frac{\Gamma_{\text {edge }, t>t_{0}}}{\Gamma_{\text {edge }, t<t_{0}}} .
$$

The error in the helium content measurement is of the order of $15 \%$ (see also appendix B) and the error in the pumping rate is of the same order (see section 2). When $R_{\text {eff }}$ and $\tau_{\text {edge }}$ are considered separately, the calibration of the influx measurement enters, which is only known to about $30 \%$. Thus, the uncertainty of a prediction of $\tau_{\mathrm{He}}^{*}$ on ITER based on our data is dominated by this measurement because in that case we are only interested in the scaling of $\tau_{\text {edge }}$ itself.

In addition to the error in the calibration, there is also an uncertainty in the validity of the estimate of the total helium influx from a main chamber, horizontal line of sight, when using equation (6). The approach we have taken can be justified for helium for two reasons. First, the mean free path of helium atoms of $0.04 \mathrm{eV}$ (which corresponds to the vessel temperature) would be $\sim 4 \mathrm{~cm}$ in a plasma with conditions as they exist on top of the edge pedestal in our experiments. Therefore, even the slowest helium atoms are expected to penetrate the SOL and we do not expect a large contribution to the observed emission to originate from the SOL. Second, it has been seen on JET that the poloidal profile of helium emission extends far outside the region close to the divertor, in contrast to that of other impurities, e.g. carbon [23]. From these emission profiles we have estimated that the influx derived from a line-of-sight in the geometric midplane is within $30 \%$ of the true volume integral. If this relationship varied strongly for the discharges in this study, specifically between ELMy H-mode and ITB discharges, our approach would not have been able to explain the difference in the measured $\tau_{\mathrm{He}}^{*} / \tau_{\mathrm{E}}^{\text {th }}$ between these regimes.

\section{Summary and outlook}

In JET ELMy H-modes with Type I ELMs and optimized pumping, the best result we achieved is $\tau_{\mathrm{He}}^{\mathrm{d} *} / \tau_{\mathrm{E}}^{\text {th }} \approx 7.2$, where $\tau_{\mathrm{He}}^{\mathrm{d} *}$ is the measured decay time of the helium content following short $100 \mathrm{~ms}$ gas puffs. The ratio worsens to $\tau_{\mathrm{He}}^{\mathrm{d} *} / \tau_{\mathrm{E}} \approx 15$ with poorer pumping in the vertical target configuration, because the effective recycling coefficient is increased in this case. The ratio is independent, over the limited range tested, of heating power. The ratio worsens with strong deuterium gas puffing, principally because $\tau_{\mathrm{E}}^{\text {th }}$ declines as the Greenwald density is approached. The value of $\tau_{\mathrm{He}}^{\mathrm{d} *}$ in L-mode and ELMy H-mode is dominated by recycling (i.e. lack of pumping).

In JET quasi-steady ITB discharges with helium beam fuelling, we find $4<\tau_{\mathrm{He}}^{\mathrm{r} *} / \tau_{\mathrm{E}}^{\text {th }}<10$, where $\tau_{\mathrm{He}}^{\mathrm{r} *}$ is the measured helium replacement time, with $\tau_{\mathrm{He}}^{\mathrm{r} *} / \tau_{\mathrm{E}}^{\text {th }} \approx 5$ obtained for the discharge with the highest value of $\beta_{\mathrm{N}}=1.4$ within the accessible operational space. These results were obtained in quasi-steady-state for a duration of up to $5 \times \tau_{\mathrm{He}}^{\mathrm{r} *}$. The value of $\tau_{\mathrm{He}}^{\mathrm{r} *}$ in these discharges is still dominated by edge transport and recycling (i.e. lack of pumping) and none of the discharges exhibit a significant increase of $\tau_{\mathrm{He}}^{\mathrm{r} *}$ due to the presence of the ITBs. The helium enrichment factor with pumping for all ITB scenarios is in the range $0.40<\eta<0.60$, which is mainly a reflection of the fact that helium is pumped, noting that without pumping, $\eta$ rises up to $\approx 0.8$.

We have shown by an independent measurement of $R_{\text {eff }}$ that it is possible to explain all observed results for $\tau_{\mathrm{He}}^{*} / \tau_{\mathrm{E}}^{\text {th }}$ in terms of the differences between the regimes in $\tau_{\text {edge }} / \tau_{\mathrm{E}}^{\mathrm{th}}$, where $\tau_{\text {edge }}$ is the helium replacement time with a source at the edge, in contrast to $\tau_{0}$, which is the replacement time with a central source. The largest value $\tau_{\text {edge }} / \tau_{\mathrm{E}}^{\text {th }} \approx 0.92$ is observed in Type I ELMy H-mode discharges, whereas in quasi-steady ITB discharges with Type III ELMs the measurements range between 0.44 and 0.58. This is comparable to L-mode where the one discharge we studied has $\tau_{\text {edge }} / \tau_{\mathrm{E}}^{\text {th }} \approx 0.50$. The properties of the SOL and the sub-divertor make a reduction of $R_{\mathrm{eff}}$ to the same value, namely 0.91 , possible in both regimes, and therefore $\tau_{\mathrm{He}}^{*} / \tau_{\mathrm{E}}^{\text {th }}$ is lower in the ITBs that we have studied. However, we do not know the contribution to $R_{\text {eff }}$ of the two factors that determine it-the return coefficient $R_{\text {ret }}$ and the fuelling efficiency, $f$, for helium, in any discharge. 
If $R_{\text {eff }}$ can be modelled for ITER, our results should allow a prediction of $\tau_{\mathrm{He}}^{*} / \tau_{\mathrm{E}}^{\text {th }}$ based on a scaling of $\tau_{\text {edge }} / \tau_{\mathrm{E}}^{\text {th }}$ between present tokamaks and ITER. Such a scaling is, however, made difficult because, to our knowledge, an independent measurement of $R_{\text {eff }}$ and $\tau_{\text {edge }} / \tau_{\mathrm{E}}^{\text {th }}$ has not been attempted as part of the helium exhaust experiments on any other tokamak and so scaling with machine size is not possible at present. Also, the errors of $R_{\text {eff }}$ and of $\tau_{\text {edge }} / \tau_{\mathrm{E}}^{\text {th }}$ are quite large (about 50\%), because the measurement relies on the absolute calibration of a vacuum UV spectrometer and the assumption that this measurement is representative for the volume source of helium. Unless inter-machine scaling of $\tau_{\text {edge }} / \tau_{\mathrm{E}}^{\text {th }}$ is a very strong function of machine geometry, such a comparison is unlikely to be conclusive.

More experiments on JET with active helium pumping are required to investigate scaling within each operating regime of $\tau_{\text {edge }} / \tau_{\mathrm{E}}^{\text {th }}$. The accuracy of such a study is much better (the relative error is about $15 \%$ ). Within the limited range studied, we have found no clear variation of $\tau_{\text {edge }} / \tau_{\mathrm{E}}^{\text {th }}$ for Type I ELMy $\mathrm{H}$-modes. Even though the two ITB regimes with LH prelude that we studied (strong ITBs at 2.63 T/2.2 MA and core ITBs at $3.45 \mathrm{~T} / 2.4 \mathrm{MA}$ with LHCD throughout the main heating phase) are characterized by different $\tau_{\text {edge }} / \tau_{\mathrm{E}}^{\text {th }}$, there are too many parameters that differ between these regimes to identify a scaling.

One further question still open is, how $\tau_{\mathrm{He}}^{*} / \tau_{\mathrm{E}}^{\text {th }}$ would behave for very high values of $\beta_{\mathrm{N}}$ at high magnetic field, i.e. for discharges like the one shown in figure 3 . We have shown that the low edge density in these scenarios is not a problem, i.e. sufficient pumping can be achieved, and our results at high values of $\beta_{\mathrm{N}}$ and low magnetic field indicate that the improvement in $\tau_{\mathrm{E}}^{\text {th }}$ combined with the intrinsically low $\tau_{\text {edge }} / \tau_{\mathrm{E}}^{\text {th }}$ (we have measured values in the range $0.10-0.25$ for the discharge shown in figure 3 ) of this type of discharge might offset any increase in $\tau_{0}$.

\section{Acknowledgments}

This paper is an extended version of a contribution to the 29th EPS Conference on Plasma Physics and Controlled Fusion held in (Montreux, Switzerland, June 2002). It includes reanalysed material from a paper by D Stork et al from the 26 th EPS Conference on Controlled Fusion and Plasma Physics (Maastricht, The Netherlands, June 1999) and a paper by K H Finken et al from the 28th EPS Conference on Controlled Fusion and Plasma Physics (Madeira, Portugal, June 2001).

This work was performed partially within the framework of the JET Joint Undertaking and partially under the European Fusion Development Agreement. It was funded in part by EURATOM, the UK Department of Trade and Industry, and the US Department of Energy under Contract No DE-AC05$00 \mathrm{OR} 22725$.

\section{Appendix A. On retention, replacement and decay time, refuelling efficiency and predictions using zero-dimensional models}

In this appendix we address the relationship between the particle retention time, $\tau^{*}$, in the case of vanishing return flux, and $\tau^{*}$ at finite return flux for particles. We will show that the

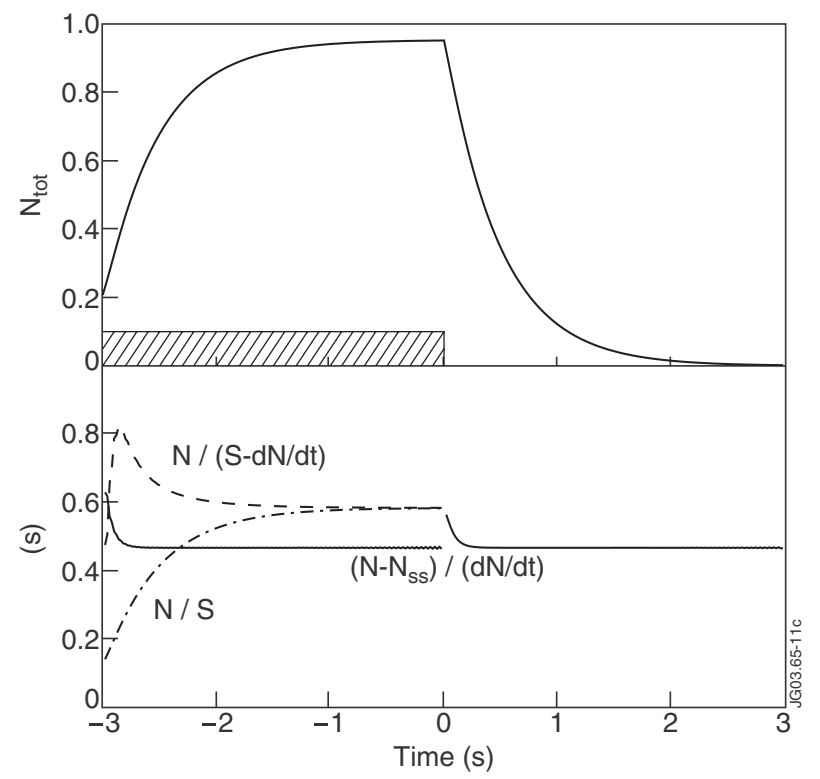

Figure 11. The upper half of the figure shows the calculated time evolution of the total number of particles, $N_{\text {tot }}$, in a cylindrical plasma of $1 \mathrm{~m}$ radius (with transport coefficients $D$ and $v$ as given in figure 12 and $R_{\text {ret }}=0.5$ ) with a phase of central deposition as indicated by the shaded area $(t<0)$ followed by a phase of decay $(t>0)$. The lower figure shows the time evolution of various characteristic time constants that could be derived from $N_{\text {tot }}$ in an experiment, as explained in the text, i.e. decay or rise time constant of the total helium content $\tau^{\mathrm{d} *}=N-N_{\mathrm{ss}} /(\mathrm{d} N / \mathrm{d} t)(-)$, replacement time $\tau^{\mathrm{r} *}=N / S(-\cdot-)$ and a variant of the latter correcting for the time evolution $\tau^{\mathrm{c}^{*}}=N /(S-\mathrm{d} N / \mathrm{dt})(---)$.

details of this relationship depend on the type of experiment that was conducted to determine $\tau^{*}$. Since these results are valid for any species, we omit the index for helium in this section.

In the first type of experiment, the decay of the total particle content, $N$, is observed once an external source of particles is turned off, and $\tau^{*}$ is identified as the rate of decay as $\tau^{\mathrm{d} *}=\left(N-N_{\mathrm{ss}}\right) /(\mathrm{d} N / \mathrm{d} t)$ where $N_{\mathrm{ss}}$ is the steady-state density at $t \rightarrow \infty$. In the second type of experiment, particles are continuously introduced in the plasma centre, and the replacement time is calculated as $\tau^{\mathrm{r} *}=N / S$, where $S$ is the total particle source rate. While the steady-state solution develops, it is also possible to try to correct for the retention of particles by calculating $\tau^{\mathrm{c}^{*}}=N /(S-\mathrm{d} N / \mathrm{d} t)$. However, this correction is incomplete, and in identical plasmas the result will be $\tau^{\mathrm{d} *} \neq \tau^{\mathrm{r} *} \neq \tau^{\mathrm{c}^{*}}$ during this transient phase, because the density profile shape is necessarily different. The actual values of $\tau^{*}$ also depend on the shape of the radial profile of the central and edge source, but this effect will not be discussed in this section.

To demonstrate this difference, we show the results of a simple calculation assuming cylindrical geometry and a plasma radius of $1 \mathrm{~m}$ in figures 11 and 12 . In this example, ions are introduced in the centre with a source rate $S_{0}$ for $t<0$. The source is then turned off for $t>0$, and the density is allowed to decay. We include an edge source (recycling flux) $S_{\text {edge }}=R_{\text {ret }} \times \Gamma_{\text {out }}$, where $R_{\text {ret }}$ is the return coefficient. The recycling flux is modelled by introducing ions inside the last closed flux surface near the plasma boundary. This allows us 

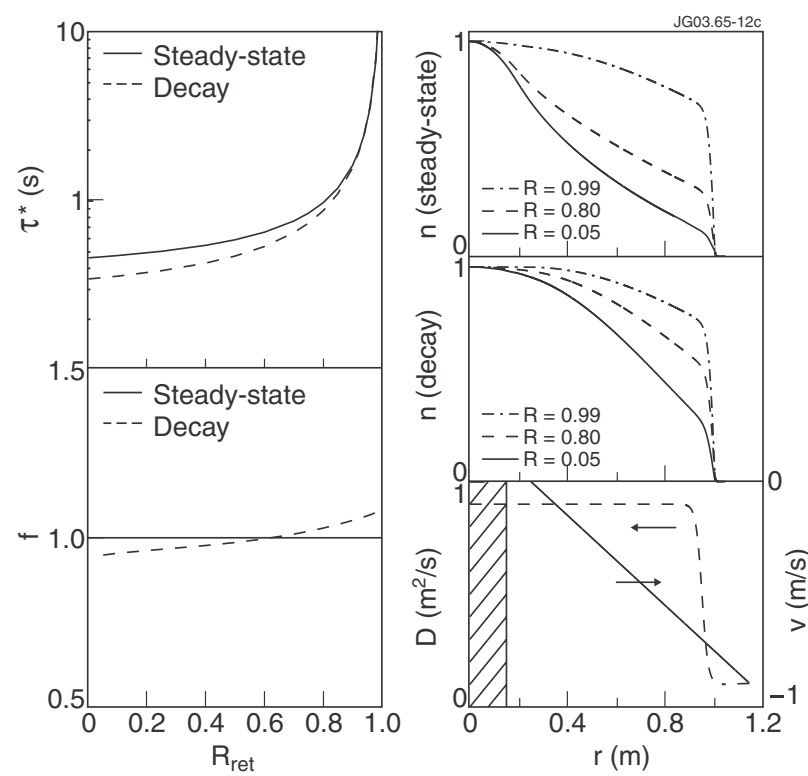

Figure 12. The right half of the figure shows (from top to bottom) the profile shape adopted in steady state and during exponential decay for various values of particle return coefficient, $R_{\text {ret }}$, and the transport coefficients used in the model calculation. The central source is located in the shaded area. The top left half of the figure shows two possible results for $\tau^{*}$, the replacement time in steady state and the time constant for exponential decay, as a function of $R_{\text {ret }}$. A fuelling efficiency, $f$, is derived from both results for $\tau^{*}$ and is shown in the bottom left half of the figure. The result is the expected value of unity only for the case of the steady-state replacement time.

to ignore all physics relating to the introduction by neutrals. Specifically, the fuelling efficiency for this type of artificial edge source is unity, as is the fuelling efficiency for the central source, thus $R_{\text {eff }}=R_{\text {ret }}$ (see equation (2)).

In the calculation the radial density profile is obtained from particle conservation as

$$
\frac{\mathrm{d} n}{\mathrm{~d} t}=-\frac{1}{r} \frac{\partial}{\partial r}\left(r\left(D \frac{\partial n}{\partial r}-v n\right)\right)+s-\frac{n}{\tau_{\|}},
$$

where a time constant for parallel losses, $\tau_{\|}$, is introduced in the SOL. This ansatz for the particle flux represents a diffusive term plus a convective term, i.e. a flux driven by gradients in other plasma quantities but the density itself.

It can be seen in figure 12 that the calculated $\tau_{0}^{\mathrm{r} *}=\mathrm{N} / \mathrm{S}_{0}$, once steady state has been reached, is always larger than $\tau_{0}^{\mathrm{d} *}=N /(\mathrm{d} N / \mathrm{d} t)$ noting that the helium content does not at first decay exponentially while the density profile shape relaxes (figure 11). It is interesting to note that the time constant for decay (the solid line just after $t=0$ in figure 12) starts off with a value equal to the steady-state result (the dashed and dashdotted lines just before $t=0$ in figure 12). Experimentally, this will be very difficult to determine, because the time window when this is the case is very short and too few data points are available to analyse. Figure 11 also illustrates that it is necessary to wait until steady state has been reached, since considering $\tau^{\mathrm{c}^{*}}=N /(S-\mathrm{d} N / \mathrm{d} t)$ results in a time dependent result. The overshoot in $\tau^{\mathrm{c}^{*}}$ occurs as long as the density profile is not yet in steady state because the particle flux through all
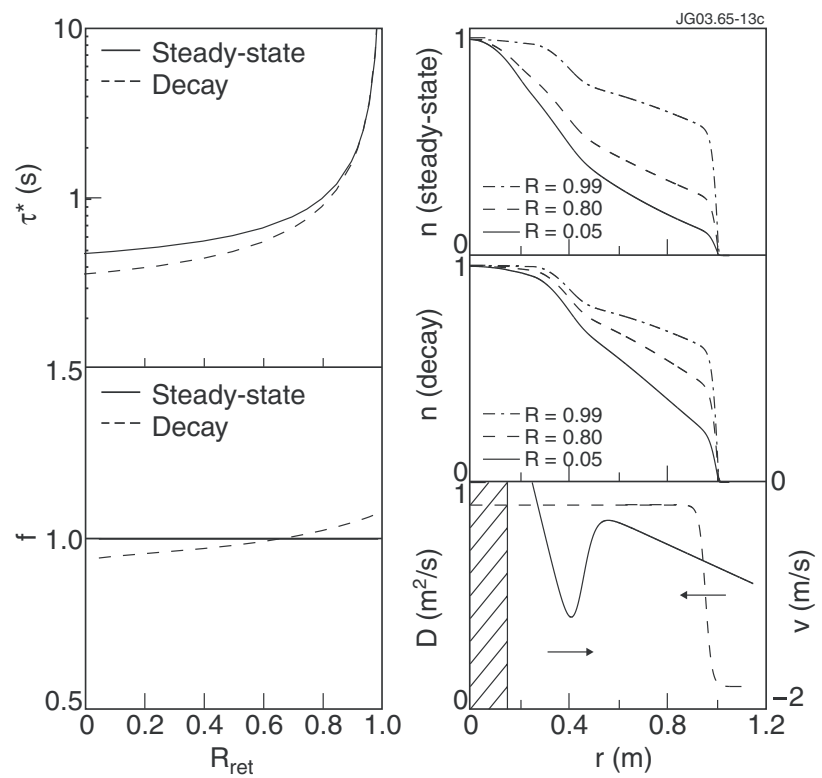

Figure 13. As figure 12 except a region of increased convective transport is introduced for $r<0.5 \mathrm{~m}$. The profile of $v$ is chosen to give the same steady-state profile shape without central source as the case illustrated in figure 14, which has reduced diffusive transport instead.

flux surfaces, including the last closed flux surface, is still increasing and is not yet equal to the central source rate.

Using equations (1) and (2) we can calculate, as a consistency check, the actual refuelling efficiency, $f$, from the solutions obtained by our calculations as

$$
f=\frac{1-R_{\mathrm{ret}}}{R_{\mathrm{ret}}} \frac{\tau^{*}-\tau_{0}}{\tau_{\text {edge }}} .
$$

In figure 12, we also show the result of this equation using the results for $\tau^{\mathrm{d} *}$ and $\tau^{\mathrm{r} *}$ from the analysis of steady state and decay. When the steady-state replacement is used we obtain $f=1$ independent of $R_{\text {ret }}$ as expected. When the decay time is used, $f$ can become larger than unity, and will depend on $R_{\text {ret }}$. The reason for this unphysical behaviour is that the radial profile in the steady-state case is a linear combination of two functions, the solution with central source only and the solution with edge source only. A solution during the decay phase, on the other hand, is not. Therefore, a linear ansatz fails.

For high values of $R_{\text {ret }}$ the difference between $\tau^{\mathrm{d} *}$ and $\tau^{\mathrm{r} *}$ is reduced (figure 12). Most of the changes occur for $R_{\text {ret }}>0.8$, although this particular value reflects the particular ratio of $\tau_{0} / \tau_{\text {edge }} \approx 3$, which in turn is determined by the profiles of $D$ and $v$ that we have chosen, in this example.

To study the sensitivity to variations in core transport coefficients, we have performed the same calculation with the transport terms modified in the centre by an additional inward drift and with a reduced diffusion coefficient. The results are shown in figures 13 and 14. These two 'enhanced core confinement' cases have been chosen to have the same solution in a source free region, which is given by

$$
\frac{1}{n} \frac{\partial n}{\partial r}=\frac{v}{D}
$$

These two calculations illustrate that the two transport coefficients have a different effect on the resulting retention 
Helium exhaust experiments on JET
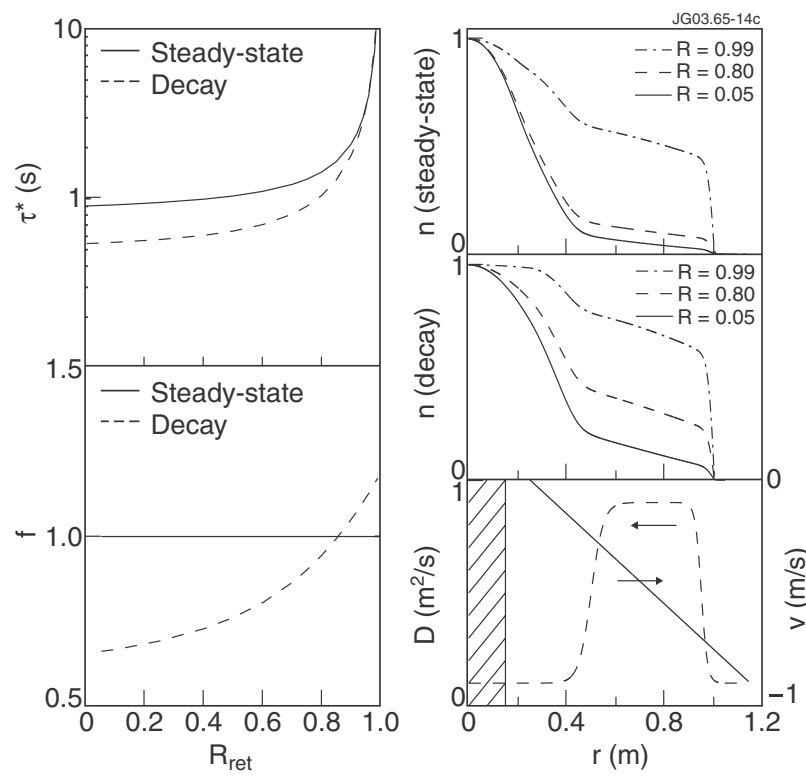

Figure 14. As figure 12 except a region of reduced diffusive transport is introduced for $r<0.5 \mathrm{~m}$. The profile of $D$ is chosen to give the same steady-state profile shape without a central source as the case illustrated in figure 13 , which has increased convective transport instead.

time in the presence of a central source. The reduced diffusion, which acts on the gradient, can result in strongly peaked density profiles, accompanied by a significant increase in $\tau^{\mathrm{d} *}$ and $\tau^{\mathrm{r} *}$ (figure 14). If the same peaking without a central source is mainly due to inward convection, this is not very efficient at retaining a central source since it acts on the density, and $\tau^{\mathrm{d} *}$ and $\tau^{\mathrm{r} *}$ are not strongly modified. The second aspect this calculation illustrates is that the effect of reduced central diffusion on $\tau^{\mathrm{r} *}$ is more pronounced than on $\tau^{\mathrm{d} *}$. As a numerical example, for $R_{\text {ret }}=0.8$, the replacement time $\tau^{\mathrm{r} *}$ increases from 0.97 to $1.01 \mathrm{~s}$ in the case with increased inward convection and to $1.42 \mathrm{~s}$ with reduced diffusion. The decay time $\tau^{\mathrm{d} *}$ increases from $0.87 \mathrm{~s}$ to $0.90 \mathrm{~s}$ and $1.04 \mathrm{~s}$, respectively. A third point worth noting is that the failure of the linear ansatz for the refuelling efficiency is larger in the case of reduced central diffusion than in the other two cases.

In summary, the steady-state replacement time $\tau^{\mathrm{r} *}$ is always longer than the decay time $\tau^{\mathrm{d} *}$ for the same plasma conditions. Only the true steady-state replacement time can be used to predict results at one specific value of $R_{\text {ret }}$ from those at a different value of $R_{\text {ret }}$ using a zero-dimensional ansatz. To do this based on experimental data, it is necessary to estimate the replacement time for an edge source, $\tau_{\text {edge }}$. Reduced central diffusion is more effective at retaining particles than increased inward convection for the same ratio of $v / D$ in the absence of a central source, with the effect more pronounced for the steady-state replacement time.

\section{Appendix B. On measurement of the decay time constant and the replacement time}

In this appendix we discuss the assumptions made and the statistical and systematic errors of the techniques adopted in

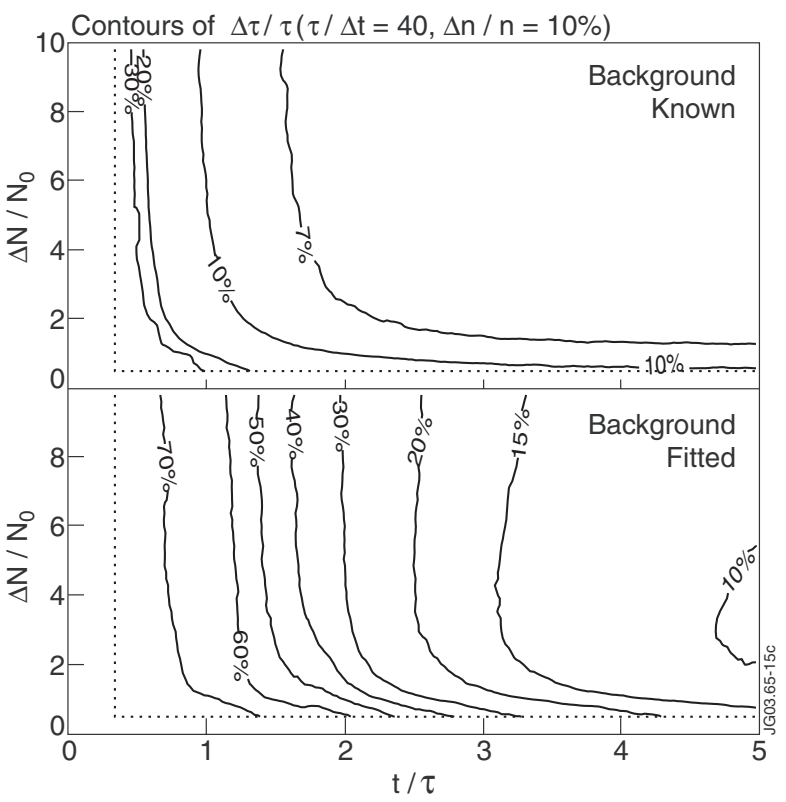

Figure 15. Contours of errors in derived decay time constant, $\tau$, as function of observed duration of decay, $t / \tau$, and amplitude over background, $\Delta N / N_{0}$. The time resolution $\Delta t$ of the signal in this example provides 40 time points per decay time with a $10 \%$ accuracy per data point. This corresponds to the actual JET experiments for $\tau=2 \mathrm{~s}$. When the background level is known, it is sufficient to observe the decay for $\frac{2}{3}$ of a decay time to achieve $20 \%$ accuracy, provided $\Delta N / N>2$. If the background level needs to be fitted, almost three decay times need to be observed.

this paper to measure the decay time constant of the helium content and the replacement time of helium.

Two parameters control the accuracy of a decay time analysis, the time period during which the decay is observed and the magnitude, $\Delta N$, of the puff. In figure 15 this is illustrated for two cases: one where the background level, $N_{0}$, is known, and one where the background level itself has to be extracted from the experiment. For the first case we need to perform a two parameter fit, for the second case a three parameter fit, to the measurement. The contours of constant error in figure 15 have been derived from generating exponential decay data, and then analysing them. Time resolution and random noise have been chosen to be representative of the JET helium diagnostic. If the background is known, a $20 \%$ accuracy for $\tau_{\mathrm{d}}$ is achieved after about $\frac{2}{3}$ of a decay time (10\% after one decay time). If the background needs to be extracted as well, 2.5 decay times have to be included, and observation during five decay times is required to achieve $10 \%$ accuracy. Since the discharge duration is limited by technical constraints, and the puff should be applied only after steady-state conditions have been achieved, it is not possible to observe the decay for long enough to perform a three parameter fit. Therefore, it is necessary to make an assumption about the helium background.

The residual helium in the experiment is due to wall storage from previous discharges [24]. In the example shown in figure 16 we can see that the helium in the plasma builds up to a steady-state level following the application of NBI heating. The heat and particle flux to the divertor and wall surfaces 


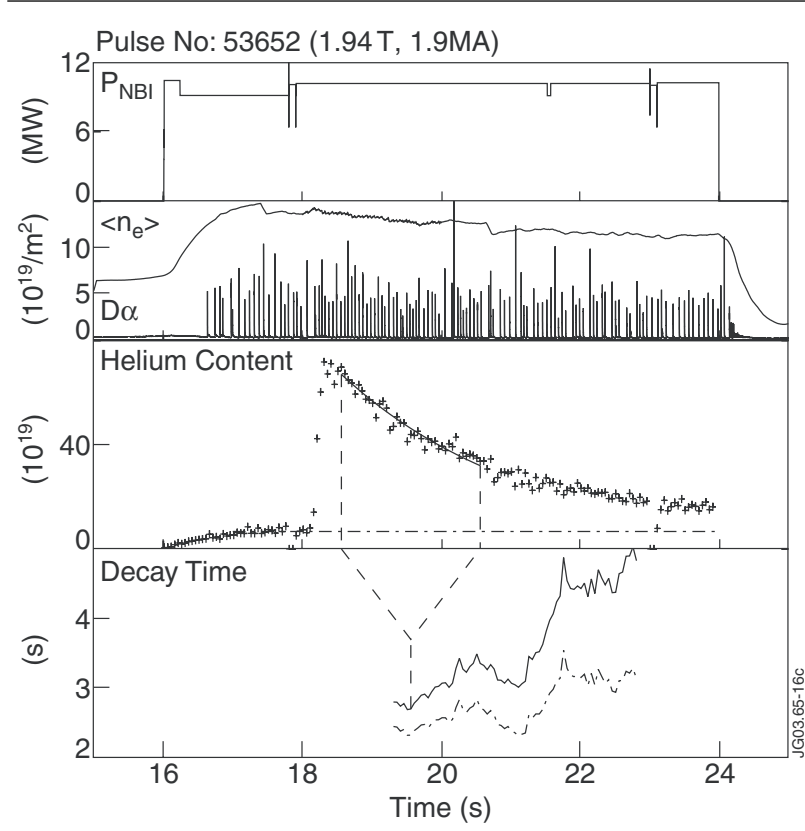

Figure 16. Analysis of exponential decay of helium content for JET-\#53652 following a gas puff at $58.0 \mathrm{~s}$. The residual helium content before the puff builds up in response to the application of NBI heating, and is due to wall storage of helium. The decay time is analysed in a floating time window of $2 \mathrm{~s}$ duration (- - - ). When it is assumed that the helium level returns to the residual level (.- - in the bottom two figures), the derived decay time rises from $2.54 \pm 0.15$ to $3.07 \pm 0.22 \mathrm{~s}$. If, instead, the analysis is performed without subtracting the residual level (_ - ), the derived decay time rises from $2.82 \pm 0.15$ to $4.71 \pm 0.22 \mathrm{~s}$. The latter method is chosen in the analysis in this paper.

releases the stored helium, and the residual level is established as equilibrium between this source and the pumping by the ArFCP. We have performed one discharge without helium puff in the ELMy H-mode series \#46514-46541, and one without helium beams in the ITB series \#53885-53932, where we have seen no further increase or indeed any reduction of the helium influx or content during $8 \mathrm{~s}$ of constant heating power. Even so, the helium content may or may not decay to the initial residual level after a puff. First, the pumping speed of the ArFCP deteriorates as deuterium and helium are trapped (see section 2), which slows down the rate of decay, but also changes the equilibrium helium content for a constant rate of release from the wall. Second, an increase of the amount of helium stored in the wall might result from the helium puff and from helium escaping from the plasma following the puff. Quantification of both of these effects is not possible by measurement, and thus requires detailed modelling, which is probably not warranted since the decay time is not actually the helium retention time, as discussed in appendix B.

A pragmatic solution, therefore, is to analyse the decay time under the assumption that the helium content will return to the residual level, but to limit the number of data to cover a floating $2 \mathrm{~s}$ time window. Alternatively, we can perform the same analysis setting $N_{0}=0$. Both methods result in a time dependent decay time constant, as illustrated in figure 16. At this point the choice between the two assumptions on $N_{0}$ becomes a matter of taste. It is, however, important in the analysis shown in section 5 to be consistent. If a finite value for $N_{0}$ is used, then the influx and pumping rate used in

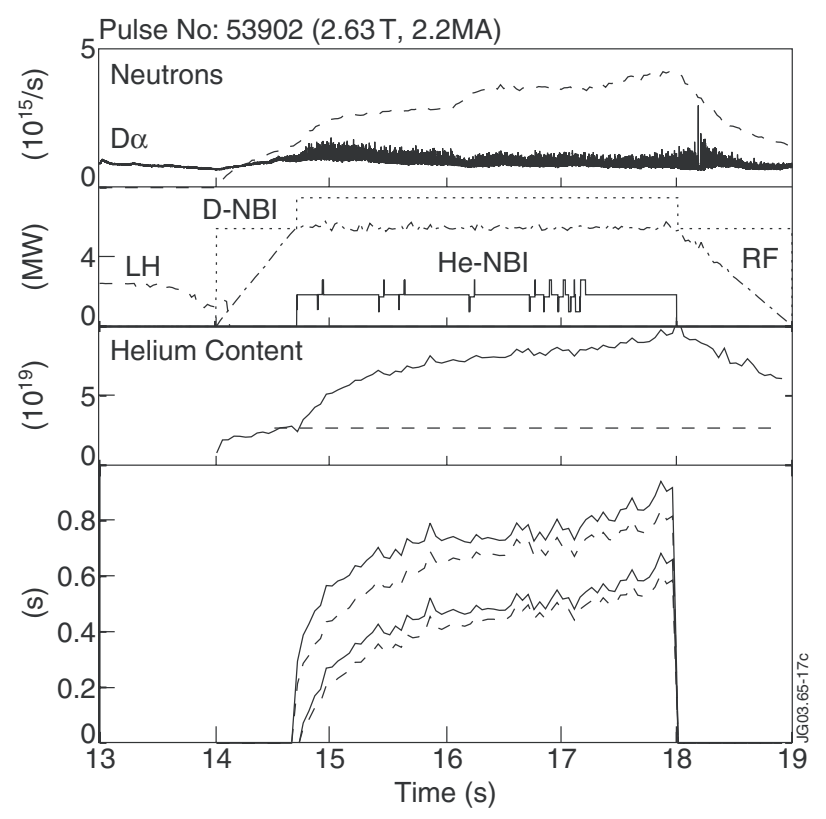

Figure 17. Analysis of helium replacement time for JET-\#53902. The dashed lines in the bottom part of the figure correspond to $\tau^{\mathrm{r} *}$ and the solid lines to $\tau^{\mathrm{c}^{*}}$ in the calculation. This correction is necessary because of the underlying evolution of the background plasma and because the pumping speed of the ArFCP is not constant for the whole duration of the discharge. If the residual helium level is subtracted to calculate an incremental helium replacement time, the result is about $0.45 \mathrm{~s}$ (lower two curves). Without subtraction, which is the method used in this paper, we obtain about $0.75 \mathrm{~s}$ (upper two curves).

equation (7) need to be corrected for the residual level as well. To simplify this analysis we have decided to set $N_{0}=0$ in this paper.

For consistency, we make the same assumption $\left(N_{0}=0\right)$ also in the calculation of the helium replacement time in experiments with a central source. In addition, it is necessary to correct for the time evolution of the helium content, i.e. to calculate $N /(S-\mathrm{d} N / \mathrm{d} t)$, since it is never truly steady state, partly because the current profile and hence the background plasma are continuously evolving even in these quasi-steady ITB discharges, but also because the pumping speed of the ArFCP decreases with time. To avoid the initial overshoot that was discussed in appendix A we wait for two replacement times before using the data point in any further analysis. An example showing four possible definitions for the replacement time is shown in figure 17. This particular discharge does not exhibit the overshoot, whereas the discharge shown in figure 7 does.

In contrast to the derivation of the decay time constant for the helium content, measurement of the replacement time requires an absolute measurement of helium content as well as knowledge of the central source. The helium density is derived from charge-exchange spectroscopy data by a self-consistent beam attenuation calculation [25]. The factor dominating the systematic error is the knowledge of the cross sections for beam attenuation and line emission. It would be realistic to assume that the overall accuracy cannot be better than $20 \%$. However, we note that, following helium gas puffs in discharges without active pumping, we find an increase of the derived helium content in the plasma that agrees with the 
amount puffed to within 5\%, which we thus take to be the error of this measurement. The shine through of the helium beams calculated for the ITB discharges studied in this paper is about $20 \%$, so that $80 \%$ is deposited in the plasma. The errors of this calculation depend mainly on the line integral density and the atomic data for beam attenuation. The error bar for this term is asymmetric. At best, all helium could be deposited in the plasma, which means there is a lower limit on the error for $\tau^{*}$ of $20 \%$ but this is too pessimistic. In combination with the error of the helium density measurement, we believe that the derived helium replacement time is accurate to about $15 \%$, i.e. the error is comparable to the error of the decay time measurements.

\section{References}

[1] Reiter D., Wolf G. and Kever H. 1990 Nucl. Fusion 302141

[2] See chapter 3, ITER Physics Basis 1999 Nucl. Fusion 39 $2420-1$

[3] Hogan J. 1997 J. Nucl. Mater. 241-243 68

[4] Wade M.R. et al 1995 Phys. Plasmas 22357

[5] Sakasai A. et al 1995 J. Nucl. Mater. 220-222 405

[6] Sakasai A. et al 1998 Proc. 17th Int. Conf. on Fusion Energy (Yokohama, 1998) (Vienna: IAEA) CD-ROM file EX6/5 and $<$ http://www.iaea.org/programmes/ripc/physics/ start.htm>

[7] Sakasai A. et al 2001 J. Nucl. Mater. 290-293957

[8] Hillis D.L. et al 1990 Phys. Rev. Lett. 652382

[9] Takenaga H. et al 1999 Nucl. Fusion 391917

[10] Kukushkin A.S. et al 2002 Nucl. Fusion 42187

[11] The JET Team (prepared by Monk R.D.) 1999 Nucl. Fusion 391751
[12] Bucalossi J. et al 2001 Proc. 28th EPS Conf. on Controlled Fusion and Plasma Physics (Funchal, Portugal, 2001) vol 25A (ECA) p 1629

[13] Groth M. et al 2002 Nucl. Fusion 42591

[14] Morgan P.D. 2001 Helium partial measurements using a Penning gauge: a new approach Proc. 6th Int. Conf. on Advanced Diagnostics for Magnetic and Inertial Fusion (Varenna, Italy, 2001) ed Peter E Stott and Alan Wootton (New York: Kluwer Academic/Plenum) ISBN 0-306-47297-X

[15] Janeschitz G. et al 1996 Proc. 16th Int. Atomic Energy Agency Conf. on Fusion Energy (Montreal, Canada) IAEA-CN-64/F2, p 759

[16] See chapter 2, ITER Physics Basis 1999 Nucl. Fusion 392175

[17] Dux R., Giroud C. and Zastrow K.-D. 2004 Nucl. Fusion 44260

[18] Mailloux J. et al 2002 Phys. Plasmas 92156

[19] Challis C.D. et al 2002 Plasma Phys. Control. Fusion 441031

[20] Behringer K., Summers H.P., Denne B., Forrest M. and Stamp M. 1989 Plasma Phys. Control. Fusion 312059

[21] ADAS, Atomic Data and Analysis Structure http://adas.phys.strath.ac.uk

[22] von Hellermann M. et al 1995 Proc. 22nd EPS Conf. on Controlled Fusion and Plasma Physics (Bournemouth, UK, 1995) vol 19C (ECA) II-009

[23] Lawson K.D. et al 2002 Proc. 29th EPS Conf. on Plasma Physics and Controlled Fusion (Montreux, Switzerland, 2002) vol 26B (ECA) p 2-040

[24] Finken K.H. et al 1990 J. Nucl. Mater. 176-177 816

[25] von Hellermann M.G. 1993 Atomic and Plasma-Material Interaction Processes in Controlled Thermonuclear Fusion ed R K Janev and H W Darwin (Amsterdam: Elsevier) 